\title{
Leakage-Based Robust Beamforming for Multi-Antenna Broadcast System With Per-Antenna Power Constraints and Quantized CDI
}

\author{
Ming Ding, Member, IEEE, Meng Zhang, Hanwen Luo, and Wen Chen, Senior Member, IEEE
}

\begin{abstract}
In this paper, we investigate the robust beamforming schemes for a multi-user multiple-input-single-output (MU-MISO) system with per-antenna power constraints and quantized channel direction information (CDI) feedback. Our design objective is to maximize the expectation of the weighted sum-rate performance by means of controlling the interference leakage and properly allocating the power among user equipments (UEs). First, we prove the optimality of the non-robust zero-forcing (ZF) beamforming scheme in the sense of generating the minimum amount of average inter-UE interference under quantized CDI. Then we derive closed-form expressions of the cumulative density function (CDF) of the interference leakage power for the non-robust $\mathrm{ZF}$ beamforming scheme, based on which we adjust the leakage thresholds and propose two robust beamforming schemes under per-antenna power constraints with an iterative process to update the per-UE power allocations using the geometric programming (GP). Simulation results show the superiority of the proposed robust beamforming schemes compared with the existing schemes in terms of the average weighted sum-rate performance.
\end{abstract}

Index Terms-Leakage, multiuser, per-antenna power constraints, quantized CDI, robust beamforming, zero-forcing.

\section{INTRODUCTION}

$\mathbf{M}$ ULTI-ANTENNA broadcast systems have gained considerable attention as they can offer both spatial multiplexing and multi-user (MU) diversity gains [1]. In the multiantenna broadcast channel (BC) model, the multiplexing gain can be achieved by simultaneously serving multiple user equipments (UEs) by space division multiple access schemes with the dirty paper coding (DPC) [2] or low-complexity linear transmit beamforming, e.g., zero-forcing (ZF) beamforming [3]. Moreover, when the UE number is large, the capacity of the $\mathrm{BC}$ system also grows with the UE number according to a double logarithm scaling law due to the MU diversity gain [4]. However, all these promising results are based on the assumption of perfect channel direction information (CDI) available at the

Manuscript received December 17, 2012; revised May 05, 2013 and July 04, 2013; accepted July 15, 2013. Date of publication July 30, 2013; date of current version September 17, 2013. The associate editor coordinating the review of this manuscript and approving it for publication was Prof. Huaiyu Dai. This work was supported by Sharp Laboratories of China Co., Ltd.

M. Ding is with Sharp Laboratories of China Co., Ltd., Shanghai 201203, China (e-mail: ming.ding@cn.sharp-world.com).

M. Zhang, H. Luo, and W. Chen are with the Department of Electronic Engineering, Shanghai Jiao Tong University, Shanghai 200240, China (e-mail: mengzhang@sjtu.edu.cn; hwluo@sjtu.edu.cn; wenchen@sjtu.edu.cn).

Digital Object Identifier 10.1109/TSP.2013.2275491 base station (BS), which is too ideal for practical systems, especially for the frequency division duplex (FDD) system such as the fourth generation $(4 \mathrm{G})$ cellular network, e.g., the long term evolution advanced (LTE-A) FDD system [5]. The imperfectness of CDI is mainly resulted from the limited-bit CDI quantization process performed by the UE [6].

The existence of CDI quantization error motivates the design of robust beamforming, which takes the uncertain channel distortions into account [7]-[19]. In [7], the authors proposed robust beamforming schemes for an MU multi-antenna BC system to minimize the BS transmission power while maintaining certain quality-of-service (QoS) requirements for the worst case model, i.e., treating channel errors as norm bounded matrices, and the stochastic model, i.e., assuming certain statistical properties of channel errors. The authors of [8] addressed a more general problem by considering additional constraints, such as keeping the interference under a preset tolerable level and individually shaping the beamforming vectors. In [9], the authors investigated robust beamforming schemes to minimize the sum of UEs' mean squared errors (MSEs). Considering inter-UE interference leakage [10], the authors of [11] designed a robust beamforming scheme to maximize a lower bound of each UE's average signal-to-leakage-plus-noise ratio (SLNR). Recently, in [12] and [13], the authors proposed another leakagebased robust transmit beamforming scheme, which optimizes the average signal-to-interference-plus-noise ratio (SINR) performance implicitly by maximizing the average signal power subject to probabilistic leakage and noise power constraints. Besides, in [14], by taking finite-rate CDI feedback into account the authors investigated the transceiver design for a two-UE multiple-input multiple-output (MIMO) interference channel, where each precoder or equalizer is divided into outer and inner parts to eliminate the cross-link interference. In [15], a joint design of the channel estimator and the quantization function was proposed based on the criterion of MSE minimization. Furthermore, robust beamforming schemes have been extended to more sophisticated models such as the MIMO relay networks [16]-[18] and the multi-cell coordinated beamforming operations [19], etc.

In this paper, we further investigate the robust beamforming design based on the approach of leakage control. In particular, we consider the optimization of the weighted sum-rate performance and more realistic power constraints, which limit the BS transmission power on a per-antenna basis. Compared with the existing robust beamforming schemes, our assumption on the transmission power is more practical since each antenna of a 


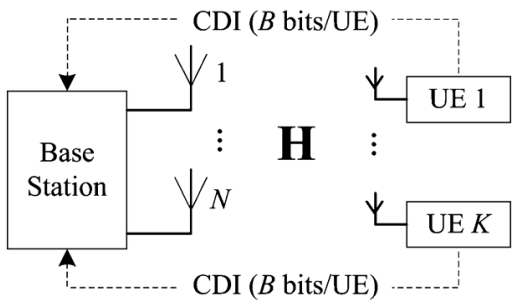

Fig. 1. Illustration of a downlink MU-MISO system with limited-bit CDI.

multi-antenna BS [20] is normally equipped with an individual power amplifier at its analog front-end. Our contributions are two folds:

1) We prove the optimality of the non-robust ZF beamforming scheme in the sense of generating the minimum amount of average inter-UE interference under limited-bit CDI, and derive closed-form expressions of the cumulative density function $(\mathrm{CDF})$ of the interference leakage power for the non-robust ZF beamforming scheme.

2) We adjust the leakage thresholds based on the derived CDF of the leakage power and propose a minimum average leakage control (MALC) and a relaxed average leakage control (RALC) beamforming schemes under per-antenna power constraints, together with an iterative process to update the power allocation among UEs using the geometric programming (GP) to maximize the weighted sumrate performance.

Although in this paper we mainly treat the MU multipleinput-single-output (MISO) model, our results can be extended to other BC models, such as MIMO relay networks [21] and multi-cell joint transmissions [22].

Notations: $(\cdot)^{\mathrm{T}},(\cdot)^{\mathrm{H}},(\cdot)^{-1},(\cdot)^{\dagger}, \operatorname{tr}\{\cdot\}$ and $\operatorname{rank}\{\cdot\}$ stand for the transpose, conjugate transpose, inverse, pseudo-inverse, trace and rank of a matrix, respectively. $\mathbf{I}_{N}$ stands for an $N \times N$ identity matrix. $\mathbf{A}_{i,:}, \mathbf{A}_{:, j}$ and $\mathbf{A}_{i, j}$ respectively denote the $i$-th row, $j$-th column and $(i, j)$-th entry of matrix $\mathbf{A}$. Besides, $\mathbf{A} \succeq$ 0 and $\mathbf{A} \in \mathbb{H}_{N}^{+}$mean that matrix $\mathbf{A}$ is positive semi-definite and $\mathbf{A}$ is an $N$ by $N$ positive semi-definite Hermitian matrix, respectively. $\|\mathbf{a}\|$ and $\mathbf{a}_{i}$ denotes the Euclidean norm and $i$-th element of vector a. $\mathbb{E}_{[\mathbf{x}]}\{\cdot\}$ and $\operatorname{Re}\{\cdot\}$ denote the expectation operation over a random vector $\mathbf{x}$ and the real part of a complex value. $\mathrm{C}_{j}^{i}$ counts the combinations of choosing $i$ elements from a set of $j$ elements. $\mathcal{N}(\mathbf{0}, \mathbf{X})$ represents a circularly symmetric complex Gaussian distribution with mean of zero vector and covariance matrix $\mathbf{X}$. Finally, we denote $\operatorname{Pr}(x)$ as the probability of event $x$.

\section{SYSTEM MODEL}

We consider a downlink MU-MISO system with limited-bit CDI feedback as shown in Fig. 1, where a BS is equipped with $N$ transmit antennas, and $K$ single-antenna UEs are served simultaneously. To support $K$ independent data streams, it requires $N \geq K$. However, our results can be easily extended to the case of $N<K$ with UE selection performed at the BS [23].

In Fig. 1, the large-scale channel attenuation coefficient and the small-scale base-band channel vector between the BS and the $k$-th $(k \in\{1,2, \ldots, K\}) \mathrm{UE}$ are denoted as $\xi_{k}$ and $\mathbf{h}_{k} \in \mathbb{C}^{1 \times N}$, respectively. Let $\mathbf{w}_{j} \in \mathbb{C}^{N \times 1}$ be the beamforming vector for UE $j$. Then the signal received at UE $k$ can be described by

$$
y_{k}=\xi_{k} \mathbf{h}_{k} \sum_{j=1}^{K} \mathbf{w}_{j} x_{j}+n_{k}=\xi_{k} \mathbf{h}_{k} \mathbf{W} \mathbf{x}+n_{k},
$$

where $\mathbf{x}=\left[x_{1}, \ldots, x_{k}, \ldots, x_{K}\right]^{\mathrm{T}}$ and $x_{k}$ is the data symbol intended for UE $k$. Without loss of generality, we assume that $\mathbf{x}$ satisfies $\mathbb{E}_{[\mathbf{x}]}\left\{\mathbf{x x}^{\mathrm{H}}\right\}=\mathbf{I}_{K}$. The channel coefficients in $\mathbf{h}_{k}$ are assumed to experience independently identical distribution (i.i.d.) Rayleigh flat fading and remain unchanged during the downlink MU-MISO transmission. $n_{k}$ is a zero-mean circularly symmetric complex Gaussian (ZMCSCG) noise variable with $\mathbb{E}_{\left[n_{k}\right]}\left\{n_{k} n_{k}^{\mathrm{H}}\right\}=N_{0}$. In addition, $\mathbf{W}=\left[\mathbf{w}_{1}, \mathbf{w}_{2}, \ldots, \mathbf{w}_{K}\right]$ and it is subject to an average per-antenna transmit power constraint expressed as

$$
\mathbb{E}_{[\mathbf{x}]}\left\{\left[\mathbf{W} \mathbf{x}(\mathbf{W} \mathbf{x})^{\mathrm{H}}\right]_{n, n}\right\}=\left[\mathbf{W} \mathbf{W}^{\mathrm{H}}\right]_{n, n} \leq P_{n},
$$

where $P_{n}$ is the maximum transmission power of the $n$-th $(n \in$ $\{1,2, \ldots, N\})$ antenna. In addition, we denote the BS's maximum transmission power as $P=\sum_{n=1}^{N} P_{n}$. By stacking the received signals of all UEs, we have

$$
\mathbf{y}=\mathbf{H W x}+\mathbf{n},
$$

where $\mathbf{y}=\left[y_{1}, y_{2}, \ldots, y_{K}\right]^{\mathrm{T}}, \mathbf{H}=\left[\xi_{1} \mathbf{h}_{1}^{\mathrm{T}}, \xi_{2} \mathbf{h}_{2}^{\mathrm{T}}, \ldots, \xi_{K} \mathbf{h}_{K}^{\mathrm{T}}\right]^{\mathrm{T}}$ and $\mathbf{n}=\left[n_{1}, n_{2}, \ldots, n_{K}\right]^{\mathrm{T}}$.

The information of $\mathbf{H}$, i.e., the channel state information (CSI), is composed of two parts, which are the channel direction information (CDI) and channel magnitude information (CMI). The CDI is the normalized base-band channel vector of UE $k$ denoted as $\tilde{\mathbf{h}}_{k}=\frac{\mathbf{h}_{k}}{\left\|\mathbf{h}_{k}\right\|}$ and the CMI is expressed by $\xi_{k}\left\|\mathbf{h}_{k}\right\|$ or $\xi_{k}^{2}\left\|\mathbf{h}_{k}\right\|^{2}$. In practice, perfect CSI is usually not available at the BS side. Hence, we first assume imperfect CDI for the interested MU-MISO system shown in Fig. 1, where each UE quantizes its CDI and feeds it back to the BS with $B$ bits. The quantized CDI is defined as the index of a vector $\hat{\mathbf{h}}_{k}$ chosen from a random vector quantization (RVQ) codebook $\mathbf{C}_{k}=\left\{\mathbf{c}_{k, 1}, \mathbf{c}_{k, 2}, \ldots, \mathbf{c}_{k, 2^{B}}\right\}$ [24] to match $\tilde{\mathbf{h}}_{k}$. To be more specific, $\mathbf{C}_{k}$ consists of $2^{B}$ unit vectors $\mathbf{c}_{k, n}$ $\left(n \in\left\{1,2, \ldots, 2^{B}\right\}\right)$ isotropically distributed in $\mathbb{C}^{1 \times M}$ and $\hat{\mathbf{h}}_{k}$ is selected as

$$
\hat{\mathbf{h}}_{k}=\underset{\mathbf{c}_{k, n} \in \mathbf{C}_{k}}{\arg \max }\left|\mathbf{c}_{k, n} \tilde{\mathbf{h}}_{k}^{\mathrm{H}}\right| .
$$

Then $\tilde{\mathbf{h}}_{k}$ can be decomposed as

$$
\tilde{\mathbf{h}}_{k}=\cos \left(\angle\left(\tilde{\mathbf{h}}_{k}, \hat{\mathbf{h}}_{k}\right)\right) \hat{\mathbf{h}}_{k}+\sin \left(\angle\left(\tilde{\mathbf{h}}_{k}, \hat{\mathbf{h}}_{k}\right)\right) \mathbf{e}_{k},
$$

where $\mathbf{e}_{k}$ is a quantization error vector orthogonal to $\hat{\mathbf{h}}_{k}$.

Regarding the CMI, $\xi_{k}$ or $\xi_{k}^{2}$ can be inferred from UE $k$ 's pathloss information, i.e., $\frac{1}{\xi_{k}^{2}}$, which is implicitly reported to the BS for mobility management in existing cellular networks [25]. In addition, $\left\|\mathbf{h}_{k}\right\|$ or $\left\|\mathbf{h}_{k}\right\|^{2}$ can be easily quantized using $M$ bits as long as its $\mathrm{CDF}$ is known. Since we consider the Rayleigh fading channels in this paper, $\left\|\mathbf{h}_{k}\right\|^{2}$ 
follows a chi-squared distribution with its $\mathrm{CDF}$ expressed as $P_{R}(r)=1-\mathrm{e}^{-r} \sum_{l=0}^{N-1} \frac{r^{l}}{l !}[26]$. Thus we can divide $P_{R}(r)$ into $2^{M}$ segments and select the midpoint of each segment to construct the codebook for the quantization of $\left\|\mathbf{h}_{k}\right\|^{2}$ as $\left\{T_{i}=P_{R}^{-1}\left(\frac{2 i+1}{2^{M+1}}\right) \mid i \in\left\{0,1, \ldots, 2^{M}-1\right\}\right\}$. Then the quantized $\left\|\mathbf{h}_{k}\right\|^{2}$ is given by

$$
\hat{A}_{k}=\underset{T_{i}}{\arg \min }\left(\left|\left\|\mathbf{h}_{k}\right\|^{2}-T_{i}\right|\right) .
$$

In the sequel, $\xi_{k}^{2}$ will be referred to as the pathloss CMI (PL-CMI) and $\left\|\mathbf{h}_{k}\right\|^{2}$ as the channel fading CMI (CF-CMI). The PL-CMI is assumed to be perfect because it changes very slowly, and hence its quantization accuracy can be consistently improved over a long period of time. The CF-CMI, on the other hand, should be subject to quantization errors since it varies as fast as the CDI. Fortunately, in our simulations, we will show that even with the average CF-CMI only, i.e., $\mathbb{E}_{\left[\mathbf{h}_{k}\right]}\left\{\left\|\mathbf{h}_{k}\right\|^{2}\right\}$, the performance of the interested beamforming schemes is comparable with that achieved by the perfect CF-CMI, i.e., $\left\|\mathbf{h}_{k}\right\|^{2}$. For notational brevity, we denote $A_{k}^{\text {ave }}=\mathbb{E}_{\left[\mathbf{h}_{k}\right]}\left\{\left\|\mathbf{h}_{k}\right\|^{2}\right\}$ hereafter. Note that $A_{k}^{\text {ave }}$ can be obtained through analytical calculation or numerical simulation based on the assumption of the channel model. Thus, $A_{k}^{\text {ave }}$ is not required to be fed back to the BS, i.e., $M=0$ for the average CF-CMI case. In the following, we will concentrate on the quantized CDI $\hat{\mathbf{h}}_{k}$ with $B>0$ and assume average CF-CMI at the BS. We will explicitly state if quantized CF-CMI $\hat{A}_{k}$ is considered.

\section{Non-RobUst BeAmForming SCHEMES}

For non-robust beamforming schemes, the channel uncertainties due to the CDI quantization errors are ignored so that at the BS side each UE's channel vector is directly replaced by $\check{\mathbf{h}}_{k}=\sqrt{A_{k}^{\text {ave }}} \hat{\mathbf{h}}_{k}$. First, we discuss the non-robust ZF beamforming scheme with per-antenna power constraints, which results will serve as the benchmark for performance comparison in our simulations.

\section{A. The Non-Robust ZF Beamforming Scheme With Per-Antenna Power Constraints}

A commonly used beamforming scheme for the downlink MU-MISO system is based on the ZF approach, which aims to completely mitigate the inter-UE interference. Under the sum power constraint, the non-robust ZF beamforming vector for $U E$ $k$ can be derived as [3]

$$
\mathbf{w}_{k}^{\mathrm{ZF}}=\sqrt{\tilde{P}_{k}} \tilde{\mathbf{w}}_{k}^{\mathrm{ZF}}=\sqrt{\tilde{P}_{k}} \frac{\check{\mathbf{H}}_{:, k}^{\dagger}}{\left\|\check{\mathbf{H}}_{:, k}^{\dagger}\right\|},
$$

where $\tilde{P}_{k}$ is the transmission power for UE $k$ and it satisfies the sum power constraint $\sum_{k=1}^{K} \tilde{P}_{k} \leq P$. Besides, $\tilde{\mathbf{w}}_{k}^{\mathrm{ZF}}$ is the normalized precoding vector and $\check{\mathbf{H}}^{\dagger}$ is the pseudo-inverse of $\check{\mathbf{H}}=\left[\xi_{1} \check{\mathbf{h}}_{1}^{\mathrm{T}}, \xi_{2} \check{\mathbf{h}}_{2}^{\mathrm{T}}, \ldots, \xi_{K} \check{\mathbf{h}}_{K}^{\mathrm{T}}\right]^{\mathrm{T}}$. In the following, the non-robust ZF beamforming scheme based on (7) will be referred to as the non-robust ZF scheme or the ZF scheme for short.

It is obvious that $\mathbf{w}_{k}^{\mathrm{ZF}}$ may violate the per-antenna power constraints shown in (2). In [27], the authors investigated the ZF beamforming with per-antenna power constraints and demonstrated that the optimal solution depends on the specific objective function. Considering the maximization of the weighted sum-rate for a MISO system, the authors of [27] addressed that the non-robust beamforming solution can be found by solving a standard semi-definite program (SDP) problem shown as

$$
\begin{aligned}
\max _{\mathbf{Q}_{k} \in \mathbb{H}_{N}^{+}} & f\left(\left\{\mathbf{Q}_{k}\right\}\right)=\sum_{k=1}^{K} \alpha_{k} \log _{2}\left(1+\frac{\xi_{k}^{2} \check{\mathbf{h}}_{k} \mathbf{Q}_{k} \check{\mathbf{h}}_{k}^{\mathrm{H}}}{N_{0}}\right) \\
\text { s.t. } \quad & \operatorname{tr}\left\{\mathbf{Q}_{k} \check{\mathbf{h}}_{j}^{\mathrm{H}} \check{\mathbf{h}}_{j}\right\}=0, \quad \forall j \neq k ; \\
& \sum_{k=1}^{K}\left[\mathbf{Q}_{k}\right]_{n, n} \leq P_{n}, \quad \forall n,
\end{aligned}
$$

where $\mathbf{Q}_{k} \in \mathbb{H}_{N}^{+}$denotes that $\mathbf{Q}_{k}$ is an $N$ by $N$ positive semidefinite Hermitian matrix. The first and second sets of constraints in problem (8) represent the requirements of zero interference among UEs and per-antenna power limitations, respectively. Problem (8) is a convex optimization problem and its numerical solution can be obtained by the use of standard mathematical softwares [28]. Note that it has been proven in [27] that problem (8) always admits a solution with rank-one matrices. Thus, the rank-one constraints on $\left\{\mathbf{Q}_{k}\right\}$ for beamforming operations have been omitted. Suppose that $\left\{\mathbf{Q}_{k}^{\mathrm{ZF}-\mathrm{PA}}\right\}$ is the solution to problem (8) and $\mathbf{Q}_{k}^{\mathrm{ZF}-\mathrm{PA}}=\mathbf{q}_{k}^{\mathrm{ZF}-\mathrm{PA}}\left(\mathbf{q}_{k}^{\mathrm{ZF}-\mathrm{PA}}\right)^{\mathrm{H}}$, then the non-robust $\mathrm{ZF}$ beamforming vector for UE $k$ under per-antenna power constraints becomes

$$
\mathbf{w}_{k}^{\mathrm{ZF}-\mathrm{PA}}=\mathbf{q}_{k}^{\mathrm{ZF}-\mathrm{PA}} .
$$

The non-robust beamforming scheme under per-antenna power constraints with the solution of (9) will be called as the ZF-PA scheme for abbreviation. It should be noted that both the ZF and ZF-PA schemes will suffer from large performance degradation due to unknown inter-UE interference resulted from inevitable CDI quantization errors [11], [13].

\section{B. The Non-Robust SLNR Beamforming Scheme With Per-UE Power Constraints}

The ZF approach merely focuses on the minimization of the inter-UE interference power, whereas the signal power usually suffers from considerable power loss due to forced vector steering away from the subspace spanned by other UEs' channel vectors. Therefore, an alternative SLNR approach has been proposed in [10], which suggests maximizing the ratio of the signal power over the interference leakage power plus the noise power. The non-robust SLNR beamforming scheme under per-UE power constraints, referred to as the SLNR scheme for short, generates the beamforming vector for UE $k$ as [10]

$$
\mathbf{w}_{k}^{\mathrm{SLNR}}=\sqrt{\tilde{P}_{k}} \mathbf{v}_{\max }\left\{\left(\frac{N_{0}}{\tilde{P}_{k}} \mathbf{I}_{N}+\overline{\mathbf{H}}_{k}^{\mathrm{H}} \overline{\mathbf{H}}_{k}\right)^{-1}\left(\xi_{k}^{2} \check{\mathbf{h}}_{k}^{\mathrm{H}} \check{\mathbf{h}}_{k}\right)\right\},
$$

where $\mathbf{v}_{\max }\{\mathbf{Q}\}$ denotes the eigen-vector associated with the largest eigen-value of matrix $\mathbf{Q}$ and $\overline{\mathbf{H}}_{k}$ is an extended channel matrix excluding $\xi_{k} \check{\mathbf{h}}_{k}$ from $\check{\mathbf{H}}$, i.e., $\check{\mathbf{H}}_{k}=\left[\xi_{1} \check{\mathbf{h}}_{1}^{\mathrm{T}}, \ldots, \xi_{k-1} \check{\mathbf{h}}_{k-1}^{\mathrm{T}}, \xi_{k+1} \check{\mathbf{h}}_{k+1}^{\mathrm{T}}, \ldots, \xi_{K} \check{\mathbf{h}}_{K}^{\mathrm{T}}\right]^{\mathrm{T}}$. 


\section{The Proposed Leakage-Based Robust Beamforming With Per-Antenna Power Constraints}

As for robust beamforming schemes, we should take the imperfect CDI into account when designing UEs' beamforming vectors, i.e., $\tilde{\mathbf{h}}_{k}$ will be considered as a random vector $\tilde{\mathbf{h}}_{k}^{\diamond}$ isotropically distributed around $\hat{\mathbf{h}}_{k}$. Similar to (5), $\tilde{\mathbf{h}}_{k}^{\diamond}$ can be written as [6]

$$
\tilde{\mathbf{h}}_{k}^{\diamond}=\sqrt{1-Z} \hat{\mathbf{h}}_{k}+\sqrt{Z} \mathbf{e}_{k}^{\diamond}
$$

where $\mathbf{e}_{k}^{\diamond}$ is isotropically distributed in the $(N-1)$-dimensional nullspace of $\hat{\mathbf{h}}_{k}$ and the random variable $Z$ is defined as $Z=$ $\sin ^{2}\left(\angle\left(\tilde{\mathbf{h}}_{k}^{\diamond}, \hat{\mathbf{h}}_{k}\right)\right)$.

Based on the idea of controlling the interference leakage to implicitly maximize UEs' SINRs [10], recently in [8], [11], [12] and [13], the authors proposed new robust beamforming schemes for MU-MISO systems under quantized CDI and per-UE power constraints. In [11], the SLNR maximization problem with regard to $\mathbf{w}_{k}$ was transformed to a Rayleigh quotient problem with a lower bound solution by utilizing Jensen's inequality to maximize each UE's average SLNR shown as

$$
\begin{aligned}
\max _{\mathbf{w}_{k}} & \frac{\mathbb{E}_{\left[\tilde{\mathbf{h}}_{j}^{\diamond} \mid \hat{\mathbf{h}}_{j} j=1,2, \ldots, K\right]}\left\{S_{k}^{\diamond}\right\}}{\mathbb{E}_{\left[\tilde{\mathbf{h}}_{j}^{\diamond} \hat{\mathbf{h}}_{j} j=1,2, \ldots, K\right]}\left\{L_{k}^{\diamond}\right\}+N_{0}} \\
\text { s.t. } & \operatorname{tr}\left\{\mathbf{w}_{k} \mathbf{w}_{k}^{\mathrm{H}}\right\} \leq \tilde{P}_{k},
\end{aligned}
$$

where the signal power $S_{k}^{\diamond}$ and leakage power $L_{k}^{\diamond}$ are defined as $S_{k}^{\diamond}=A_{k}^{\text {ave }}\left|\xi_{k} \tilde{\mathbf{h}}_{k}^{\diamond} \mathbf{w}_{k}\right|^{2}$ and $L_{k}^{\diamond}=\sum_{j=1, j \neq k}^{K} A_{j}^{\text {ave }}\left|\xi_{j} \tilde{\mathbf{h}}_{j}^{\diamond} \mathbf{w}_{k}\right|^{2}$, respectively. For brevity, we omit the subscription $\left[\tilde{\mathbf{h}}_{j}^{\diamond} \mid \hat{\mathbf{h}}_{j} j=\right.$ $1,2, \ldots, K]$ of $\mathbb{E}$ hereafter. According to [11], $\mathbb{E}\left\{S_{k}^{\diamond}\right\}$ can be computed as

$$
\mathbb{E}\left\{S_{k}^{\diamond}\right\}=\xi_{k}^{2} A_{k}^{\text {ave }} \mathbf{w}_{k}^{\mathrm{H}} \mathbf{U}_{k} \mathbf{w}_{k},
$$

where $\mathbf{U}_{k}=\left(1-\frac{N \eta}{N-1}\right) \hat{\mathbf{h}}_{k}^{\mathrm{H}} \hat{\mathbf{h}}_{k}+\frac{\eta}{N-1} \mathbf{I}_{N}$ and $\eta$ is computed as $\eta=2^{B} \beta\left(2^{B}, \frac{N}{N-1}\right)[6]$. Here, $\beta(x, y)$ is the beta function defined as $\beta(x, y)=\frac{\Gamma(x) \Gamma(y)}{\Gamma(x+y)}$ [29], where $\Gamma(\cdot)$ denotes the gamma function [29]. It should be noted that $\mathbf{U}_{k}$ is positive definite because it is easy to verify

$$
1-\frac{N \eta}{N-1}>0, \quad \text { for } N>1, \quad B \geq 0 .
$$

Similar to (13), $\mathbb{E}\left\{L_{k}^{\diamond}\right\}$ can be derived as

$$
\mathbb{E}\left\{L_{k}^{\diamond}\right\}=\mathbf{w}_{k}^{\mathrm{H}} \overline{\mathbf{U}}_{k} \mathbf{w}_{k},
$$

where $\overline{\mathbf{U}}_{k}=\sum_{j=1, j \neq k}^{K} \xi_{j}^{2} A_{j}^{\text {ave }} \mathbf{U}_{j}$. Then the solution to problem (12) can be written in a similar expression as in (10) with $\check{\mathbf{H}}_{k}^{\mathrm{H}} \check{\mathbf{H}}_{k}$ and $\check{\mathbf{h}}_{k}^{\mathrm{H}} \check{\mathbf{h}}_{k}$ respectively replaced by $\overline{\mathbf{U}}_{k}$ and $A_{k}^{\text {ave }} \mathbf{U}_{k}$ [11]. The scheme based on problem (12) is named the average SLNR (ASLNR) scheme by the authors of [11].

In [8], the optimization of SLNR was interpreted as keeping the expectation of leakage power below a threshold while maximizing the expectation of signal power. The optimization problem with regard to $\mathbf{w}_{k}$ can be formulated as

$$
\begin{array}{ll}
\max _{\mathbf{w}_{k}} & \mathbb{E}\left\{S_{k}^{\diamond}\right\} \\
\text { s.t. } & \mathbb{E}\left\{L_{k}^{\diamond}\right\} \leq \gamma_{k}, \text { and } \operatorname{tr}\left\{\mathbf{w}_{k} \mathbf{w}_{k}^{\mathrm{H}}\right\} \leq \tilde{P}_{k},
\end{array}
$$

where $\gamma_{k}$ is a design parameter of the leakage power threshold. Problem (16) is a non-convex quadratically constrained quadratic program (QCQP) problem [30]. However, it can be transformed to an equivalent SDP problem as

$$
\begin{aligned}
\max _{\mathbf{Q}_{k} \in \mathbb{H}_{N}^{+}} & \operatorname{tr}\left\{\mathbf{Q}_{k} \mathbf{U}_{k}\right\} \\
\text { s.t. } & \operatorname{tr}\left\{\mathbf{Q}_{k} \overline{\mathbf{U}}_{k}\right\} \leq \gamma_{k}, \text { and } \operatorname{tr}\left\{\mathbf{Q}_{k}\right\} \leq \tilde{P}_{k} ; \\
& \operatorname{rank}\left\{\mathbf{Q}_{k}\right\}=1 .
\end{aligned}
$$

Problem (17) is still a non-convex problem due to the nonconvex rank-one constraint. Applying the SDP relaxation technique [30] by omitting the rank-one constraint, we get

$$
\begin{aligned}
\max _{\mathbf{Q}_{k} \in \mathbb{H}_{N}^{+}} & \operatorname{tr}\left\{\mathbf{Q}_{k} \mathbf{U}_{k}\right\} \\
\text { s.t. } & \operatorname{tr}\left\{\mathbf{Q}_{k} \overline{\mathbf{U}}_{k}\right\} \leq \gamma_{k}, \text { and } \operatorname{tr}\left\{\mathbf{Q}_{k}\right\} \leq \tilde{P}_{k} .
\end{aligned}
$$

Now problem (18) is a standard convex SDP problem and can be solved efficiently using the mathematical software package [28]. According to [8], the solution of an SDP problem like problem (18) is always rank-one if it has at most three constraints. Since problem (18) has only two constraints, we can conclude that problem (17) and (18) are equivalent. Thus the solution for the original problem (16) can be extracted from the solution to problem (18).

Obviously, in problem (16) $\gamma_{k}$ should be carefully chosen to make sure that the leakage power constraints are neither too tight nor too loose to achieve a good balance between the maximization of $\mathbb{E}\left\{S_{k}^{\diamond}\right\}$ and the minimization of $\mathbb{E}\left\{L_{k}^{\diamond}\right\}$. It is worthwhile to note that recently in [12] and [13], the authors proposed a probabilistic approach in the control of interference leakage, i.e., to keep the event of large leakage below a certain probability. The scheme will be referred to as the probabilistic leakage control (PLC) scheme hereafter. In the PLC scheme, the leakage constraint in problem (16) is regulated as

$$
\operatorname{Pr}\left(\mathbb{E}\left\{L_{k}^{\diamond}\right\} \geq \gamma_{k}\right) \leq p_{k},
$$

where $p_{k}$ is a given probability of the event that $\mathbb{E}\left\{L_{k}^{\diamond}\right\}$ exceeds $\gamma_{k}$. According to [12] and [13], by invoking Markov's inequality, (19) can be nicely tightened by a new constraint $\mathbb{E}\left\{L_{k}^{\diamond}\right\} \leq p_{k} \gamma_{k}$, which generates a new problem shown as

$$
\begin{array}{ll}
\max _{\mathbf{w}_{k}} & \mathbb{E}\left\{S_{k}^{\diamond}\right\} \\
\text { s.t. } & \mathbb{E}\left\{L_{k}^{\diamond}\right\} \leq p_{k} \gamma_{k}, \text { and } \operatorname{tr}\left\{\mathbf{w}_{k} \mathbf{w}_{k}^{\mathrm{H}}\right\} \leq \tilde{P}_{k} .
\end{array}
$$

Problem (20) is essentially equivalent to problem (16) if we define $p_{k} \gamma_{k}$ as a new threshold $\tilde{\gamma}_{k}$. Therefore, the issue still remains regarding the appropriate proposal of $\gamma_{k}$. 


\section{A. Minimum Average Leakage Power}

In order to get some insights on the design of $\gamma_{k}$, we will start with the comparison between the average leakage power in problem (16) and that of the non-robust ZF beamforming given by (7). Our result is summarized in Theorem 1 .

Theorem 1: Let $\mathbf{w}_{k}(k \neq j)$ be a general beamforming vector expressed as $\mathbf{w}_{k}=\sqrt{\tilde{P}_{k}} \tilde{\mathbf{w}}_{k}$, where $\tilde{\mathbf{w}}_{k}$ is the normalized vector of $\mathbf{w}_{k}$. Then for arbitrary CF-CMI $A_{j}$, the expected interference leakage from UE $k$ to $j$ is lower bounded by

$$
\begin{aligned}
\mathbb{E}\left\{\left|\xi_{j} \sqrt{A_{j}} \tilde{\mathbf{h}}_{j}^{\diamond} \mathbf{w}_{k}\right|^{2}\right\} & \geq \mathbb{E}\left\{\left|\xi_{j} \sqrt{A_{j}} \tilde{\mathbf{h}}_{j}^{\diamond} \mathbf{w}_{k}^{\mathrm{ZF}}\right|^{2}\right\} \\
& =\frac{\tilde{P}_{k} \xi_{j}^{2} A_{j} \eta}{(N-1)} .
\end{aligned}
$$

Proof: See Appendix I.

From Theorem 1, we can state that the non-robust ZF beamforming scheme is optimal in the sense of generating the minimum amount of average inter-UE interference under limited-bit CDI. In other words, the average leakage power of the non-robust ZF beamforming scheme can serve as a lower bound for the interference leakage in problem (16).

\section{B. The Proposed Minimum Average Leakage Control Beamforming With Per-UE Power Constraints}

According to Theorem 1, under the assumptions of average CF-CMI and the Rayleigh channel fading, we can set the leakage power threshold $\gamma_{k}$ to the derived lower bound as

$$
\gamma_{k}^{\mathrm{MALC}}=\frac{\tilde{P}_{k} N \eta}{(N-1)} \sum_{j=1, j \neq k}^{K} \xi_{j}^{2},
$$

where $A_{j}$ in Theorem 1 has been replaced by $A_{j}^{\text {ave }}=N$, considering that for Rayleigh fading channels $\left\|\mathbf{h}_{j}\right\|^{2}$ follows a chi-squared distribution with $2 N$ degrees of freedom and its mean is $N$ [26]. On the other hand, when the BS has quantized CF-CMI, we can substitute $A_{j}$ with $\hat{A}_{j}$ and choose $\gamma_{k}^{\text {MALC }}$ as

$$
\gamma_{k}^{\mathrm{MALC}}=\frac{\tilde{P}_{k} \eta}{(N-1)} \sum_{j=1, j \neq k}^{K} \xi_{j}^{2} \hat{A}_{j}
$$

Then we can re-write problem (16) as

$$
\begin{array}{ll}
\max _{\mathbf{w}_{k}} & \mathbb{E}\left\{S_{k}^{\diamond}\right\} \\
\text { s.t. } & \mathbb{E}\left\{L_{k}^{\diamond}\right\} \leq \gamma_{k}^{\mathrm{MALC}} \text {, and } \operatorname{tr}\left\{\mathbf{w}_{k} \mathbf{w}_{k}^{\mathrm{H}}\right\} \leq \tilde{P}_{k} .
\end{array}
$$

The beamforming scheme based on problem (24) will be called the minimum average leakage control (MALC) scheme since it controls the expected leakage power according to the minimum average leakage given by Theorem 1 .

\section{The Proposed Relaxed Average Leakage Control Beamforming With Per-UE Power Constraints}

From Theorem 1, we have found the minimum threshold value for the average interference leakage shown in $(22) /(23)$. Next, we want to raise the leakage threshold so that the weighted sum-rate can be increased. The intuition is that interference minimization, e.g., ZF precoding, is generally not the optimal strategy for throughput maximization or MSE minimization. Allowing some more interference leakage can increase the weighted sum-rate due to signal power boosting. Of course if the tolerated leakage is set to be too large, the rate will inevitably decrease. Our intention is to find an appropriate leakage level, which can generate a good weighted sum-rate performance.

Under the assumption of average CF-CMI, we propose that the upper half segment of the CDF of $\left|\xi_{j} \sqrt{A_{j}^{\diamond}} \tilde{\mathbf{h}}_{j}^{\diamond} \mathbf{w}_{k}^{\mathrm{ZF}}\right|^{2}$ should serve as the analytical reference for determining how large $\gamma_{k}$ should be, where $A_{j}^{\diamond}$ is the randomly reconstructed CF-CMI at the BS based on $A_{j}^{\text {ave }}$ and the channel-fading model. Our proposal is based on the fact that the CDF of $\left|\xi_{j} \sqrt{A_{j}^{\diamond}} \tilde{\mathbf{h}}_{j}^{\diamond} \mathbf{w}_{k}^{\mathrm{ZF}}\right|^{2}$ contains tractable information on the average leakage level of a conservative beamformer $\mathbf{w}_{k}^{\mathrm{ZF}}$ with minimum generation of inter-UE interference. As a result, $\gamma_{k}^{\text {MALC }}$ will be loosened to another threshold $\gamma_{k}^{\text {RALC }}$ and we can design a relaxed average leakage control (RALC) beamforming scheme with per-UE power constraints. Since $\left|\xi_{j} \sqrt{A_{j}^{\diamond}} \tilde{\mathbf{h}}_{j}^{\diamond} \mathbf{w}_{k}^{Z \mathrm{~F}}\right|^{2}=\tilde{P}_{k} \xi_{j}^{2}\left|\sqrt{A_{j}^{\diamond}} \tilde{\mathbf{h}}_{j}^{\diamond} \tilde{\mathbf{w}}_{k}^{\mathrm{ZF}}\right|^{2}$, we can derive the CDF of $\left|\sqrt{A_{j}^{\diamond}} \tilde{\mathbf{h}}_{j}^{\diamond} \tilde{\mathbf{w}}_{k}^{\mathrm{ZF}}\right|^{2}$ and scale it by a factor of $\tilde{P}_{k} \xi_{j}^{2}$ to obtain the $\mathrm{CDF}$ of $\left|\xi_{j} \sqrt{A_{j}^{\diamond}} \tilde{\mathbf{h}}_{j}^{\diamond} \mathbf{w}_{k}^{\mathrm{ZF}}\right|^{2}$. In the following we present our result on the CDF of $\left|\sqrt{A_{j}^{\diamond}} \tilde{\mathbf{h}}_{j}^{\diamond} \tilde{\mathbf{w}}_{k}^{\mathrm{ZF}}\right|^{2}$ for Rayleigh fading channels in Theorem 2 .

Theorem 2: Let $D=\left|\sqrt{A_{j}^{\diamond}} \tilde{\mathbf{h}}_{j}^{\diamond} \tilde{\mathbf{w}}_{k}^{\mathrm{ZF}}\right|^{2}$. Then for Rayleigh fading channels the CDF of $D$ is shown in (25),

$$
\begin{aligned}
& P_{D}(d)=1+\frac{\mathrm{e}^{-d}}{(N-2) !}\left\{\sum_{n=0}^{N-2} \sum_{m=1}^{2^{B}} \mathrm{C}_{N-2}^{n} \mathrm{C}_{2^{B}} \frac{(-1)^{n+m} m}{m N-(m+n)}\right. \\
& \left.\times \sum_{l=0}^{N-1-n} l ! \mathrm{C}_{N-1-n}^{l} d^{N-1-l}\right\} \\
& -\frac{\mathrm{e}^{-d}}{(N-2) !}\left\{\sum_{n=0}^{N-2} \mathrm{C}_{N-2}^{n} \mathrm{C}_{2^{B}}^{1} \frac{(-1)^{n+1}}{N-1-n} d^{N-1}\right\} \\
& -\frac{\mathrm{e}^{-d}}{(N-2) !}\left\{\sum_{n=0}^{N-2} \sum_{m=2}^{2^{B}} \mathrm{C}_{N-2}^{n} \mathrm{C}_{2^{B}}^{m} \frac{(-1)^{n+m} m}{m N-(m+n)}\right. \\
& \left.\times\left[\sum_{l=1}^{(m-1)(N-1)-1} \frac{(-1)^{l-1} d^{N-1+l}}{l ! \mathrm{C}_{(m-1)(N-1)-1}^{l}}\right]\right\} \\
& -\frac{\mathrm{E}_{1}(d)}{(N-2) !}\left\{\sum_{n=0}^{N-2} \sum_{m=2}^{2^{B}} \mathrm{C}_{N-2}^{n} \mathrm{C}_{2^{B}} \frac{(-1)^{n+m} m}{m N-(m+n)}\right. \\
& \left.\times \frac{(-1)^{(m-1)(N-1)-1} d^{m(N-1)}}{((m-1)(N-1)-1) !}\right\} \text {. }
\end{aligned}
$$

where $\mathrm{E}_{1}(x)=\int_{x}^{\infty} \frac{\mathrm{e}^{-t}}{t} d t$ is the exponential integral function [29].

Proof: See Appendix II.

If the BS has quantized CF-CMI $\hat{A}_{j}$, then $A_{j}^{\diamond}$ should take the value of $\hat{A}_{j}$. Consequently, we can get the CDF of $\left|\xi_{j} \sqrt{\hat{A}_{j}} \tilde{\mathbf{h}}_{j}^{\diamond} \mathbf{w}_{k}^{\mathrm{ZF}}\right|^{2}$ by scaling that of $\left|\tilde{\mathbf{h}}_{j}^{\diamond} \tilde{\mathbf{w}}_{k}^{\mathrm{ZF}}\right|^{2}$ by a factor of 
$\tilde{P}_{k} \xi_{j}^{2} \hat{A}_{j}$. In Theorem 3, we show our result on the CDF of $\left|\tilde{\mathbf{h}}_{j}^{\diamond} \tilde{\mathbf{w}}_{k}^{\mathrm{ZF}}\right|^{2}$.

Theorem 3: Let $V=\left|\tilde{\mathbf{h}}_{j}^{\diamond} \tilde{\mathbf{w}}_{k}^{\mathrm{ZF}}\right|^{2}$. Then the CDF of $V$ is

$$
\begin{aligned}
& P_{V}(v)=1+(N-1) \\
& \quad \times \sum_{n=0}^{N-2} \sum_{m=1}^{2^{B}} \mathrm{C}_{N-2}^{n} \mathrm{C}_{2^{B}}^{m}(-1)^{n+m} m \frac{v^{n}-v^{m(N-1)}}{m N-(m+n)} .
\end{aligned}
$$

Proof: See Appendix III.

Based on Theorem 2 or 3, we can relax the control target of the interference leakage to the $\delta_{k}$ percent point of the leakage power of the non-robust ZF beamforming scheme. To be more specific, considering average CF-CMI, we can set $\gamma_{k}^{\text {RALC }}$ according to Theorem 2 as

$$
\gamma_{k}^{\mathrm{RALC}}=\tilde{P}_{k} \sum_{j=1, j \neq k}^{K} \xi_{j}^{2} P_{D}^{-1}\left(\delta_{k}\right),
$$

where $P_{D}^{-1}\left(\delta_{k}\right)=\underset{d}{\arg }\left\{P_{D}(d)=\delta_{k}\right\}$. When quantized $\mathrm{CF}-\mathrm{CMI}$ is available at the $\mathrm{BS}$, we can recall Theorem 3 and choose $\gamma_{k}^{\mathrm{RALC}}$ as

$$
\gamma_{k}^{\mathrm{RALC}}=\tilde{P}_{k} \sum_{j=1, j \neq k}^{K} \xi_{j}^{2} \hat{A}_{j} P_{V}^{-1}\left(\delta_{k}\right),
$$

where $P_{V}^{-1}\left(\delta_{k}\right)=\arg \left\{P_{V}(v)=\delta_{k}\right\}$. Although there is no closed-form expression for the computation of $P_{D}^{-1}\left(\delta_{k}\right)$ or $P_{V}^{-1}\left(\delta_{k}\right)$, it can be conveniently found by the bisection method for a given $\delta_{k}$ since $P_{D}(d)$ or $P_{V}(v)$ is a bounded and monotonically increasing function. In the bisection method, considering $P_{D}(d)$ as an example, first we choose a small value $d_{1}$ and a large one $d_{2}$ to construct an interval $\left[P_{D}\left(d_{1}\right), P_{D}\left(d_{2}\right)\right]$ that contains $\delta_{k}$, then we continually compare $P_{D}\left(\frac{d_{1}+d_{2}}{2}\right)$ with $\delta_{k}$ and update $d_{1}$ or $d_{2}$ with $\frac{d_{1}+d_{2}}{2}$ on condition that $\delta_{k}$ stays inside the interval $\left[P_{D}\left(d_{1}\right), P_{D}\left(d_{2}\right)\right]$. The bisection searching stops when $\left[P_{D}\left(d_{1}\right), P_{D}\left(d_{2}\right)\right]$ is sufficiently narrow, and then we can obtain $\delta_{k}=\frac{d_{1}+d_{2}}{2}$. As for the choice of $\delta_{k}$ in the proposed RALC scheme, since we want to get a relaxed leakage threshold compared with $\gamma_{k}^{\mathrm{MALC}}$, it is intuitive to look beyond $\gamma_{k}^{\text {MALC }}$ and hinge the leakage power threshold to some point on the upper half segment of $P_{D}(d)$ or $P_{V}(v)$, i.e.,

$\delta_{k} \in \begin{cases}\left(P_{D}\left(\frac{\gamma_{k}^{\mathrm{MALC}}}{\widetilde{P}_{k} \sum_{j \neq k} \xi_{j}^{2}}\right), 1\right), & \text { for } \gamma_{k}^{\mathrm{MALC}} \text { from (22); } \\ \left(P_{V}\left(\frac{\gamma_{k}^{\mathrm{MALC}}}{\tilde{P}_{k} \sum_{j \neq k} \xi_{j}^{2} \hat{A}_{j}}\right), 1\right), & \text { for } \gamma_{k}^{\mathrm{MALC}} \text { from (23). }\end{cases}$

Based on (27) or (28), the original problem (16) becomes

$$
\begin{array}{ll}
\max _{\mathbf{w}_{k}} & \mathbb{E}\left\{S_{k}^{\diamond}\right\} \\
\text { s.t. } & \mathbb{E}\left\{L_{k}^{\diamond}\right\} \leq \gamma_{k}^{\text {RALC }}, \text { and } \operatorname{tr}\left\{\mathbf{w}_{k} \mathbf{w}_{k}^{\mathrm{H}}\right\} \leq \tilde{P}_{k} .
\end{array}
$$

The beamforming scheme based on problem (29) will be referred to as the relaxed average leakage control (RALC) scheme because it relaxes the leakage threshold compared with the MALC scheme. It should be noted that though $\delta_{k}$ is a design parameter chosen to allow more interference leakage, the leakage threshold can be immediately found in an efficient way once the system parameters $\left\{N, K, B, \delta_{k}\right\}$ are provided. On the other hand, the PLC scheme [12], [13] employs an empirical method to determine the leakage threshold, which needs to exhaustively search the appropriate leakage threshold by a great amount of simulations for each parameter set $\{N, K, B\}$.

\section{The Proposed Robust Beamforming Schemes With Per-Antenna Power Constraints}

Problems (24) and (29) with the proposed leakage power thresholds are subject to power constraints on a per-UE basis. However, the appropriate choices of $\tilde{P}_{k} \mathrm{~s}$ for those problems that can optimize the system performance measure, e.g., the weighted sum-rate, under per-antenna power constraints are still unclear. In this paper, we propose a two-stage algorithm to alternately update the per-UE power allocations and beamforming vectors in order to maximize the expected weighted sum-rate performance under per-antenna power constraints.

Suppose that the precoding matrix in the $l$-th step is $\mathbf{W}^{(l)}=\left[\sqrt{\tilde{P}_{1}^{(l)}} \tilde{\mathbf{w}}_{1}^{(l)}, \sqrt{\tilde{P}_{2}^{(l)}} \tilde{\mathbf{w}}_{2}^{(l)}, \ldots, \sqrt{\tilde{P}_{K}^{(l)}} \tilde{\mathbf{w}}_{K}^{(l)}\right]$, where $\tilde{P}_{k}^{(l)}$ and $\tilde{\mathbf{w}}_{k}^{(l)}$ are respectively the beamforming power and normalized beamforming vector for UE $k$. Then the straightforward way to optimize the expected weighted sum-rate is to find new $\tilde{P}_{k}^{(l+1)}$ and $\tilde{\mathbf{w}}_{k}^{(l+1)}$ that can maximize $f\left(\mathbf{W}^{(l+1)}\right)=\mathbb{E}\left\{\sum_{k=1}^{K} \alpha_{k} \log _{2}\left(1+\frac{S_{k}^{\diamond(l+1)}}{I_{k}^{\diamond(l+1)}+N_{0}}\right)\right\}$, where $S_{k}^{\diamond(l+1)}$ and $I_{k}^{\diamond(l+1)}$ are computed as $S_{k}^{\diamond(l+1)} \quad=\quad A_{k}^{\text {ave }}\left|\xi_{k} \tilde{\mathbf{h}}_{k}^{\diamond} \sqrt{\tilde{P}_{k}^{(l+1)}} \tilde{\mathbf{w}}_{k}^{(l+1)}\right|^{2} \quad$ and $I_{k}^{\diamond(l+1)}=\sum_{j=1, j \neq k}^{K} A_{k}^{\text {ave }}\left|\xi_{k} \tilde{\mathbf{h}}_{k}^{\diamond} \sqrt{\tilde{P}_{j}^{(l+1)}} \tilde{\mathbf{w}}_{j}^{(l+1)}\right|^{2}$, respectively.

1) Updating Per-UE Powers: First, we concentrate on the update of $\widetilde{\mathbf{P}}^{(l+1)}=\left(\tilde{P}_{1}^{(l+1)}, \tilde{P}_{2}^{(l+1)}, \ldots, \tilde{P}_{K}^{(l+1)}\right)$ with the objective to maximize $f\left(\mathbf{W}^{(l+1)}\right)$ on condition of $\tilde{\mathbf{w}}_{k}^{(l+1)}=\tilde{\mathbf{w}}_{k}^{(l)}$. However, $f\left(\mathbf{W}^{(l+1)}\right)$ is hard to handle because it has no explicit expression. Instead, we treat $\left.\tilde{f}\left(\mathbf{W}^{(l+1)}\right)\right|_{\tilde{\mathbf{w}}_{k}^{(l+1)}=\tilde{\mathbf{w}}_{k}^{(l)}}$, where $\tilde{f}\left(\mathbf{W}^{(l+1)}\right)=\sum_{k=1}^{K} \alpha_{k} \log _{2}\left(1+\frac{\mathbb{E}\left\{S_{k}^{\diamond(l+1)}\right\}}{\mathbb{E}\left\{I_{k}^{\diamond(l+1)}\right\}+N_{0}}\right)$, as an approximated metric of the weighted sum-rate. In a similar way as in (13), we can obtain

$$
\mathbb{E}\left\{\left.S_{k}^{\diamond(l+1)}\right|_{\tilde{\mathbf{w}}_{k}^{(l+1)}=\tilde{\mathbf{w}}_{k}^{(l)}}\right\}=\tilde{P}_{k}^{(l+1)} \lambda_{k, k}^{(l)},
$$

where $\lambda_{k, k}^{(l)}=\xi_{k}^{2} A_{k}^{\text {ave }} \tilde{\mathbf{w}}_{k}^{(l) \mathrm{H}} \mathbf{U}_{k} \tilde{\mathbf{w}}_{k}^{(l)}$. Also we can get

$$
\mathbb{E}\left\{\left.I_{k}^{\diamond(l+1)}\right|_{\tilde{\mathbf{w}}_{k}^{(l+1)}=\tilde{\mathbf{w}}_{k}^{(l)}}\right\}=\sum_{j=1, j \neq k}^{K} \tilde{P}_{j}^{(l+1)} \lambda_{j, k}^{(l)},
$$

where $\lambda_{j, k}^{(l)}=\xi_{k}^{2} A_{k}^{\text {ave }} \tilde{\mathbf{w}}_{j}^{(l) \mathrm{H}} \mathbf{U}_{k} \tilde{\mathbf{w}}_{j}^{(l)}$. Note that $\lambda_{j, k}^{(l)}>0$ because of (14). Then with some mathematical manipulation, the optimization problem to maximize $\left.\tilde{f}\left(\mathbf{W}^{(l+1)}\right)\right|_{\tilde{\mathbf{w}}_{k}^{(l+1)}=\tilde{\mathbf{w}}_{k}^{(l)}}$ with leakage control and per-antenna power constraints can be formulated as 


$$
\begin{aligned}
\min _{\widetilde{\mathbf{P}}^{(l+1)}} & \prod_{k=1}^{K}\left(\frac{\sum_{j=1, j \neq k}^{K} \tilde{P}_{j}^{(l+1)} \lambda_{j, k}^{(l)}+N_{0}}{\sum_{j=1}^{K} \tilde{P}_{j}^{(l+1)} \lambda_{j, k}^{(l)}+N_{0}}\right)^{\alpha_{k}} \\
\text { s.t. } \quad & \mathbb{E}\left\{\left.L_{k}^{\diamond(l+1)}\right|_{\tilde{\mathbf{w}}_{k}^{(l+1)}=\tilde{\mathbf{w}}_{k}^{(l)}}\right\} \leq \gamma_{k}^{(l)}, \quad \forall k ; \\
& {\left[\sum_{k=1}^{K} \tilde{P}_{k}^{(l+1)} \tilde{\mathbf{w}}_{k}^{(l)} \tilde{\mathbf{w}}_{k}^{(l) \mathrm{H}}\right]_{n, n} \leq P_{n}, \quad \forall n, } \\
& \tilde{P}_{k}^{(l+1)} \geq 0, \quad \forall k,
\end{aligned}
$$

where $\mathbb{E}\left\{\left.L_{k}^{\diamond(l+1)}\right|_{\tilde{\mathbf{w}}_{k}^{(l+1)}=\tilde{\mathbf{w}}_{k}^{(l)}}\right\}=\sum_{j=1, j \neq k}^{K} \tilde{P}_{k}^{(l+1)} \lambda_{k, j}^{(l)}$ and $\gamma_{k}^{(l)}$ is the proposed leakage power threshold in the $l$-th step and it can be updated based on $\gamma_{k}^{\mathrm{MALC}}$ or $\gamma_{k}^{\mathrm{RALC}}$ with $\tilde{P}_{k}$ replaced by $\tilde{P}_{k}^{(l)}$ in $(22) /(23)$ or in $(27) /(28)$. Besides, the second set of constraints reflects the per-antenna power control requirement. Unfortunately, problem (32) is non-convex, and searching of the global optimal solution is of high-complexity. To our best knowledge, till now geometric programming (GP) is one of the most efficient methods to solve this specific power allocation problem [31]. According to [31], the denominator of the objective function in problem (32) can be lower-bounded by the geometric inequality shown as

$$
\prod_{k=1}^{K}\left(\sum_{j=1}^{K} \tilde{P}_{j}^{(l+1)} \lambda_{j, k}^{(l)}+N_{0}\right)^{\alpha_{k}} \geq \prod_{k=1}^{K} \prod_{j=0}^{K}\left(\frac{m_{j, k}}{\mu_{j, k}}\right)^{\mu_{j, k} \alpha_{k}}
$$

where $m_{j, k}=\left\{\begin{array}{ll}N_{0}, & j=0 \\ \tilde{P}_{j}^{(l+1)} \lambda_{j, k}^{(l)}, & j=1,2, \ldots, K\end{array}\right.$ and $\mu_{j, k}=$ $\frac{m_{j, k}}{\sum_{j=1}^{K} m_{j, k}}$. Substituting the denominator of the objective function in problem (32) with the right-hand side in (33), we can obtain a standard GP problem formulated as

$$
\begin{aligned}
\min _{\widetilde{\mathbf{P}}^{(l+1)}} & \frac{\prod_{k=1}^{K}\left(\sum_{j=0, j \neq k}^{K} m_{j, k}\right)^{\alpha_{k}}}{\prod_{k=1}^{K} \prod_{j=0}^{K}\left(\frac{m_{j, k}}{\mu_{j, k}}\right)^{\mu_{j, k} \alpha_{k}}} \\
\text { s.t. } & \frac{\mathbb{E}\left\{\left.L_{k}^{\diamond(l+1)}\right|_{\left.\tilde{\mathbf{w}}_{k}^{(l+1)}=\tilde{\mathbf{w}}_{k}^{(l)}\right\}} ^{\gamma_{k}^{(l)}} \leq 1, \quad \forall k ;\right.}{} \\
& \frac{\left[\sum_{k=1}^{K} \tilde{P}_{k}^{(l+1)} \tilde{\mathbf{w}}_{k}^{(l)} \tilde{\mathbf{w}}_{k}^{(l) \mathrm{H}}\right]_{n, n} \leq 1, \quad \forall n,}{P_{n}} \\
& \tilde{P}_{k}^{(l+1)} \geq 0, \quad \forall k .
\end{aligned}
$$

Problem (34) can be solved iteratively by using the mathematical software [28] to obtain $\widetilde{\mathbf{P}}^{(l+1)}$ followed by updating $\left\{\mu_{j, k}\right\}$ to form the new optimization problem (34) regarding $\widetilde{\mathbf{P}}^{(l+1)}$ [31]. The iteration is terminated when the power difference metric PD_metric $=\frac{\left|\widetilde{\mathbf{P}}_{(i)}^{(l+1)}-\widetilde{\mathbf{P}}_{(i-1)}^{(l+1)}\right|}{\left|\widetilde{\mathbf{P}}_{(i-1)}^{(l+1)}\right|}$ falls below a pre-determined threshold $\epsilon$, where $i$ is the iteration index in the GP algorithm. Note that according to [31], the GP algorithm can handle problem (34) very efficiently and output a nearly-optimal solution to the original problem (32).

2) Updating Per-UE Beamforming Vectors: Next, we fix $\widetilde{\mathbf{P}}^{(l+1)}$ and update $\left\{\mathbf{w}_{k}^{(l+1)}\right\}$. Here, we propose to add per-an- tenna power constraints into problem (24) and (29), and optimize the beamforming vectors in an order according to the order of $\tilde{P}_{k}^{(l+1)}$. To be more specific, suppose that $\left\{\tilde{P}_{k}^{(l+1)}\right\}$ is arranged in a descending order as $\tilde{P}_{\pi(1)}^{(l+1)} \geq \tilde{P}_{\pi(2)}^{(l+1)} \geq \ldots \geq$ $\tilde{P}_{\pi(K)}^{(l+1)}$, then $\mathbf{w}_{\pi(k)}^{(l+1)}$ shall be the $k$-th beamforming vector for optimization and the power of the $n$-th antenna should be lower than the per-antenna power headroom left by the previous $k-1$ UEs, which is

$$
u_{n, \pi(k)}^{(l+1)}=P_{n}-\sum_{i=1}^{k-1}\left[\mathbf{w}_{\pi(i)}^{(l+1)} \mathbf{w}_{\pi(i)}^{(l+1) \mathrm{H}}\right]_{n, n} .
$$

The philosophy behind our design is that, a larger $\tilde{P}_{k}^{(l+1)}$ generally indicates greater significance of treating the beamforming vector of $\mathrm{UE} k$ in the maximization of the weighed sum-rate, and hence $\mathbf{w}_{k}^{(l+1)}$ should be optimized sequentially according to the order $\pi(k)$. Based on the above discussion, we can re-formulate problem (24) and (29) as

$$
\begin{aligned}
\max _{\mathbf{w}_{\pi(k)}^{(l+1)}} & \mathbb{E}\left\{S_{\pi(k)}^{\diamond(l+1)}\right\} \\
\text { s.t. } & \mathbb{E}\left\{L_{\pi(k)}^{\diamond(l+1)}\right\} \leq \gamma_{\pi(k)}^{(l+1)} ; \\
& \operatorname{tr}\left\{\mathbf{w}_{\pi(k)}^{(l+1)} \mathbf{w}_{\pi(k)}^{(l+1) \mathrm{H}}\right\} \leq \tilde{P}_{\pi(k)}^{(l+1)} ; \\
& {\left[\mathbf{w}_{\pi(k)}^{(l+1)} \mathbf{w}_{\pi(k)}^{(l+1) \mathrm{H}}\right]_{n, n} \leq u_{n, \pi(k)}^{(l+1)}, \quad \forall n, }
\end{aligned}
$$

where $\gamma_{\pi(k)}^{(l+1)}$ is computed in a similar way as $\gamma_{k}^{(l)}$ with $\tilde{P}_{k}^{(l)}$ replaced by $\tilde{P}_{\pi(k)}^{(l+1)}$. In order to solve problem (36), we transform it to an SDP problem similar to problem (17), and then we apply the SDP relaxation technique [30] by dropping the rank-one constraints so as to get the following convex SDP problem.

$$
\begin{aligned}
\max _{\mathbf{Q}_{\pi(k)}^{(l+1)} \in \mathbb{H}_{N}^{+}} & \operatorname{tr}\left\{\mathbf{Q}_{\pi(k)}^{(l+1)} \mathbf{U}_{\pi(k)}\right\} \\
\text { s.t. } & \operatorname{tr}\left\{\mathbf{Q}_{\pi(k)}^{(l+1)} \overline{\mathbf{U}}_{\pi(k)}\right\} \leq \gamma_{\pi(k)}^{(l+1)} ; \\
& \operatorname{tr}\left\{\mathbf{Q}_{\pi(k)}^{(l+1)}\right\} \leq \tilde{P}_{\pi(k)}^{(l+1)} ; \\
& {\left[\mathbf{Q}_{\pi(k)}^{(l+1)}\right]_{n, n} \leq u_{n, \pi(k)}^{(l+1)}, \quad \forall n . }
\end{aligned}
$$

Problem (37) has $N+2$ constraints, which is larger than three for meaningful cases with $N>1$. Thereby, it is not guaranteed that the solution $\mathbf{Q}_{\pi(k)}^{(l+1)}$ is always rank-one [8]. Here we resort to the randomization technique [30] and obtain an approximate solution with rank-one matrix. Denote the solution to problem (37) as $\mathbf{Q}_{\pi(k)}^{(l+1) *}$ and suppose that $\operatorname{rank}\left\{\mathbf{Q}_{\pi(k)}^{(l+1) *}\right\}>1$, then we generate a random vector $\tilde{\mathbf{q}}_{\pi(k)}^{(l+1)} \sim \mathcal{N}\left(\mathbf{0}, \mathbf{Q}_{\pi(k)}^{(l+1) *}\right)$ and scale it by a factor $\rho$ to make sure that all the constraints in problem (37) are satisfied, i.e., $\mathbf{q}_{\pi(k)}^{(l+1)}=\rho \tilde{\mathbf{q}}_{\pi(k)}^{(l+1)}$. The vector randomization process is repeated by $L_{\text {rand }}$ times and we select the vector with the largest performance measure for problem (37) as the solution to problem (36), i.e.,

$$
\mathbf{w}_{\pi(k)}^{(l+1)}=\underset{\mathbf{q}_{\pi(k)}^{(l+1),(i)}, i \in\left\{1,2, \ldots, L_{\text {rand }}\right\}}{\arg \max }\left(\operatorname{tr}\left\{\mathbf{Q}_{\pi(k)}^{(l+1),(i)} \mathbf{U}_{\pi(k)}\right\}\right),
$$


where $\mathbf{Q}_{\pi(k)}^{(l+1),(i)}=\mathbf{q}_{\pi(k)}^{(l+1),(i)}\left(\mathbf{q}_{\pi(k)}^{(l+1),(i)}\right)^{\mathrm{H}}$.

3) The Proposed Iterative Algorithm: With the updated $\mathbf{w}_{\pi(k)}^{(l+1)}$, we can compute $\tilde{f}\left(\mathbf{W}^{(l+1)}\right)$ as the performance measure for the $(l+1)$-th step. In order to find a locally optimal solution, the two-stage algorithm can be iteratively operated with $L_{\text {algo1 }}$ times and the beamforming vectors associated with the largest performance measure will be output as the final solutions. The proposed robust beamforming with per-antenna power constraints based on threshold $\gamma_{k}^{\mathrm{MALC}}$ or $\gamma_{k}^{\mathrm{RALC}}$ will be respectively referred to as the MALC-PA or RALC-PA scheme in the following, which is summarized in Algorithm 1. In Algorithm 1, we initialize the beamforming vectors as those of the ZF-PA scheme for simplicity.

Algorithm 1 The MALC-PA or RALC-PA scheme

Result: $\left\{\mathbf{w}_{k}^{\text {algo1 }}\right\}$

1 Initialize $\mathbf{w}_{k}^{\text {algo1 }}=\mathbf{w}_{k}^{\mathrm{ZF}-\mathrm{PA}}(k \in\{1,2, \ldots, K\})$ using (9);

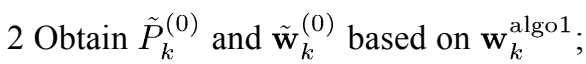

3 Set perf_metric $=0$;

4 for $l:=0$ to $L_{\text {algol }}$ do

5 Fix $\tilde{\mathbf{w}}_{k}^{(l)}$ and update $\tilde{P}_{k}^{(l+1)}$ by solving problem (34);

6 Sort $\tilde{P}_{k}^{(l+1)}$ in a descendant order $\pi(k)$;

7 Fix $\tilde{P}_{k}^{(l+1)}$ and update $\mathbf{w}_{\pi(k)}^{(l+1)}$ by solving problem (37);

8 Compute the performance measure $\tilde{f}\left(\mathbf{W}^{(l+1)}\right)$;

9 If $\tilde{f}\left(\mathbf{W}^{(l+1)}\right)>$ perf_metric then

$10 \quad$ Update perf_metric $=\tilde{f}\left(\mathbf{W}^{(l+1)}\right)$;

$11 \quad$ Update $\mathbf{w}_{k}^{\text {algo1 }}=\mathbf{w}_{k}^{(l+1)},(k \in\{1,2, \ldots, K\})$;

$12 l=l+1$;

\section{Simulation Results and Discussions}

In this section, we present simulation results to compare the performance of the interested beamforming schemes. In our simulations, the system parameters are configured as $(N, K)=(4,4),\left\{\alpha_{k}\right\}=\left[\frac{3}{2}, \frac{3}{2}, 1,1\right], \xi_{k}=1$ and $P_{k}=\frac{P}{N}$. In the proposed MALC-PA and RALC-PA schemes, $\delta_{k}$ equals to 0.8 for the computation of $\gamma_{k}^{\mathrm{MALC}}$ and $\gamma_{k}^{\text {RALC }}$. Besides, $L_{\text {rand }}=1000, \epsilon=0.01$ and $L_{\text {algo } 1}=1 \sim 10$. Moreover, we define the BS's SNR as $S N R=P / N_{0}$. All channels are assumed to experience uncorrelated Rayleigh fading and the entries of $\mathbf{h}_{k}$ are i.i.d. ZMCSCG random variables with unit variance. The results are averaged over 5,000 independent channel realizations.

\section{A. Verifications of Theorem 2 and 3}

Before discussing the performance of various beamforming schemes, in Fig. 2 and 3 we respectively compare the numerical results of $P_{D}(d)$ and $P_{V}(v)$ with our analytical results in Theorem 2 and 3 , when $(N, B)=(2,2),(2,4),(4,4)$ or $(4,6)$. As

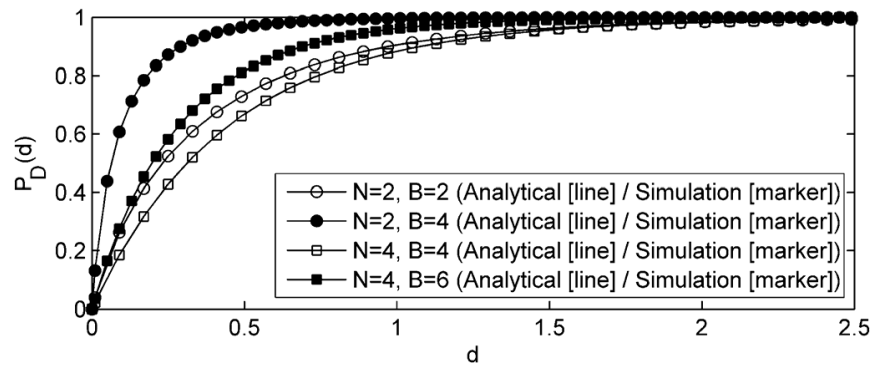

Fig. 2. Simulation and analytical results of $P_{D}(d)$ with different $(N, B)$.

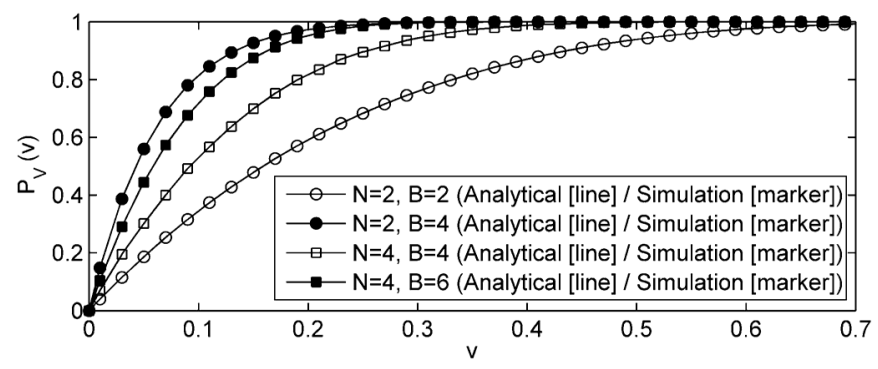

Fig. 3. Simulation and analytical results of $P_{V}(v)$ with different $(N, B)$.

can be seen from Fig. 2 and 3, the simulation results perfectly agree with our analysis, which provides theoretical foundations for the parameter configuration of $\gamma_{k}^{\mathrm{MALC}}$ and $\gamma_{k}^{\mathrm{RALC}}$ in the proposed MALC-PA and RALC-PA schemes.

\section{B. Average Weighted Sum-Rate Performance With Per-UE Power Constraints}

In this sub-section, we compare the average weighted sum-rate performance of the ZF, SLNR, ASLNR, PLC schemes, together with the proposed MALC and RALC schemes since they are all based on per-UE power constraints. For simplicity, equal power allocation among UEs i.e., $\tilde{P}_{k}=\frac{P}{K}$, is employed. Besides, we assume average CF-CMI and 6-bit CDI. For the PLC scheme, $\gamma_{k}$ and $p_{k}$ are respectively set to 0.9 and 0.05 as in [13]. The performance is exhibited in Fig. 4, from which we can see that the ZF scheme gives the performance lower bound and the SLNR scheme performs rather poorly in high SNR regime while the ASLNR scheme manages to recover a large portion of the performance loss for the SLNR scheme. Though the PLC scheme exhibits comparable performance with the proposed MALC scheme when the SNR is high, the proposed MALC and RALC schemes significantly outperform the PLC scheme in low to medium SNR regimes, which shows the great significance of appropriate choices of the leakage threshold. It is interesting to note that the proposed MALC scheme performs better than the proposed RALC scheme in high SNR regime since inter-UE interference dominates the performance when the SNR is large and the strategy of minimizing the average leakage prevails. The opposite observation can be drawn for low SNR regime. It should also be noted that although the proposed MALC and RALC schemes only show comparable performance with the ASLNR scheme respectively in high and low SNR regimes, they are more flexible in handling the per-antenna power constraints, which leads to the proposed 


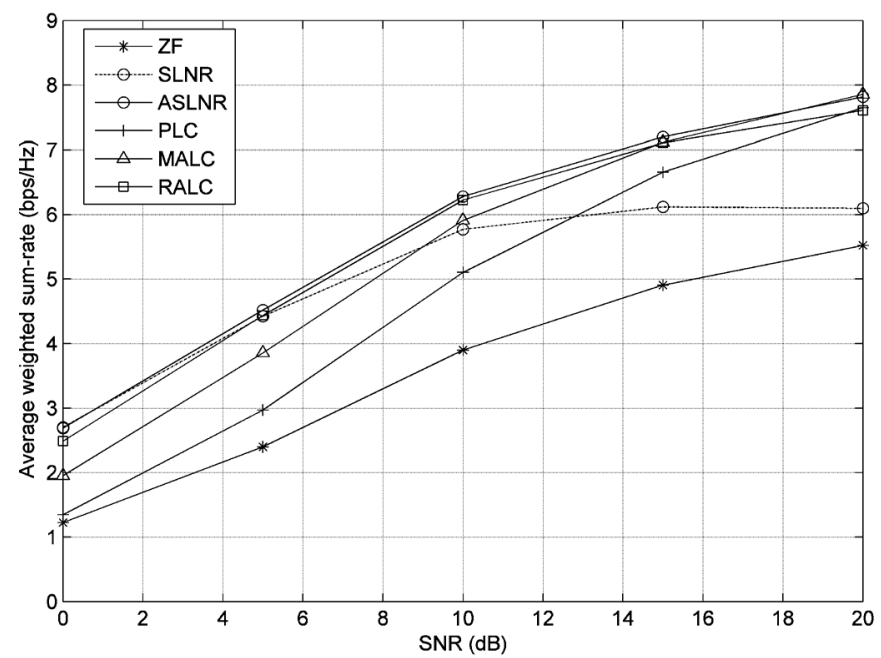

Fig. 4. Average weighted sum-rate performance with per-UE power constraints.

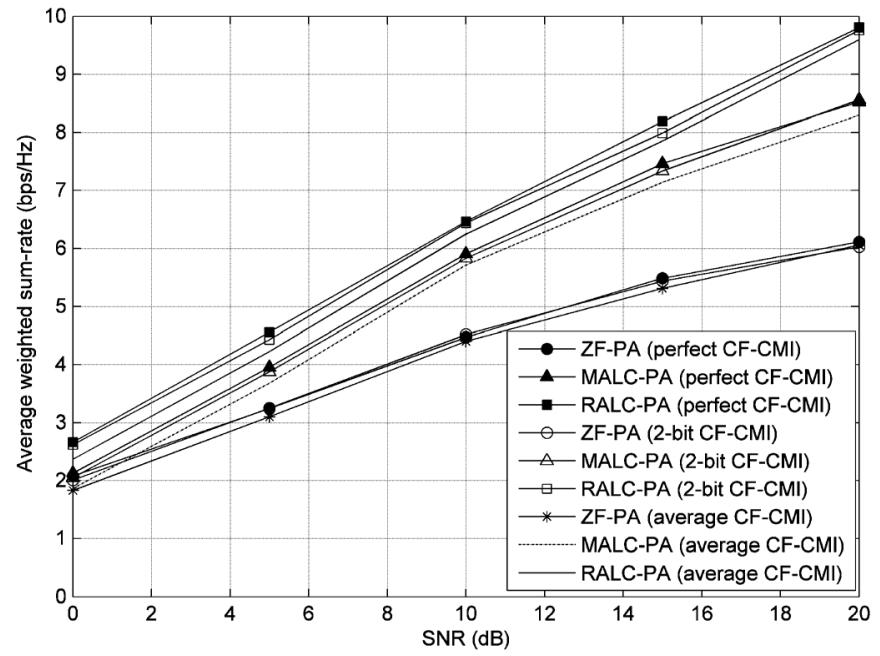

Fig. 5. Performance comparison with different bits of CF-CMI quantization.

MALC-PA and RALC-PA schemes to be evaluated in the following sub-sections.

\section{Impact of CF-CMI Quantization on the Performance}

The average weighted sum-rate performance with different bits of CF-CMI quantization is provided in Fig. 5 for the ZF-PA scheme and the proposed MALC-PA and RALC-PA schemes to show the impact of CF-CMI quantization on the system performance. We assume $L_{\text {algo1 }}=10,6$-bit CDI and average/2$\mathrm{bit} /$ perfect CF-CMI in our simulations. For the perfect CF-CMI case, $A_{k}^{\text {ave }}$ is replaced by $\left\|\mathbf{h}_{k}\right\|^{2}$ in corresponding formulations. As can be seen from Fig. 5, the performance curves of the average CF-CMI are very close to those of the perfect CF-CMI. Besides, the 2-bit quantized CF-CMI is sufficient to achieve almost the same performance as the perfect CF-CMI. Considering the minor effectiveness of quantizing the CF-CMI on the performance, we only consider the average CF-CMI case in the following simulations.

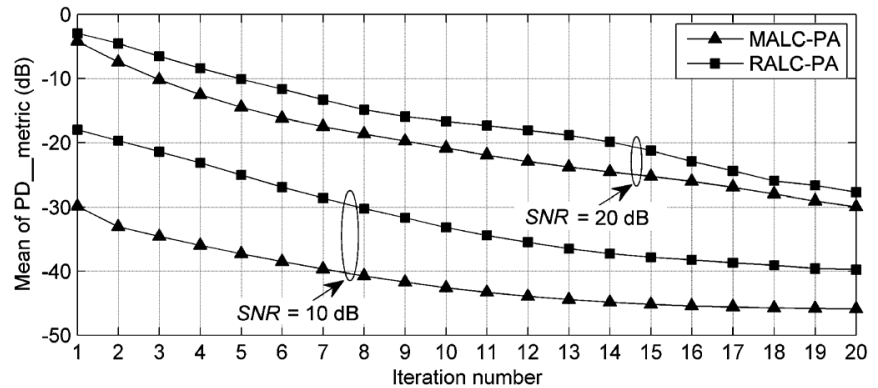

Fig. 6. Convergence of the power updating based on GP.

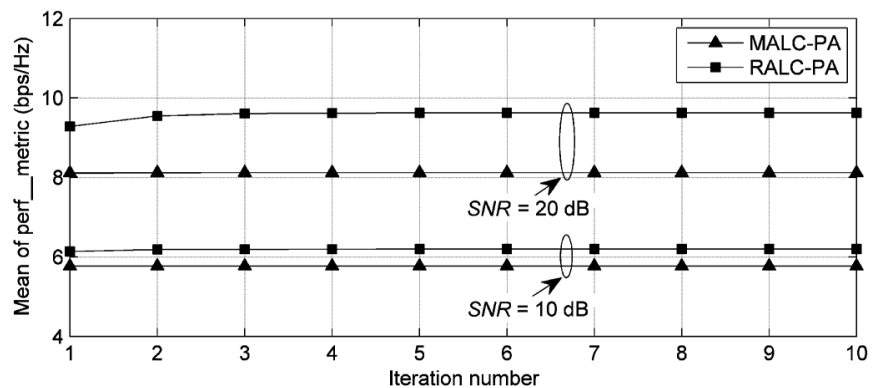

Fig. 7. Convergence of the Algorithm 1.

\section{Convergence of the Proposed Algorithm}

In this sub-section, we investigate the convergence behavior of the proposed Algorithm 1. We assume average CF-CMI, 6-bit $\mathrm{CDI}$ and $S N R=10$ or $20 \mathrm{~dB}$. First, we check the convergence of the power updating based on the GP algorithm addressed in Section IV-D. The mean value of $P D \_$metric when $L_{\text {algol }}=1$ is plotted in $\mathrm{dB}$ scale in Fig. 6, from which we can find that the per-UE power allocation coverges rapidly, e.g., only $5 \sim 15$ iterations are needed for PD_metric $<\epsilon$ depending on the working SNR.

Next, we investigate the convergence of the proposed Algorithm 1. In Fig. 7, we plot the mean value of perf_metric defined in Algorithm 1 when $L_{\text {algo } 1} \in\{1,2, \ldots, 10\}$. As can be seen from Fig. 7, both the MALC-PA and RALC-PA schemes quickly converge to their final solutions after merely 2 or 3 iterations, which makes the proposed beamforming schemes feasible for practical uses.

\section{E. Average Weighted Sum-Rate Performance With Per-Antenna Power Constraints}

In Fig. 8, we show the average weighted sum-rate performance with per-antenna power constraints of the proposed schemes with $L_{\text {algo1 }}=3$ as well as the ZF-PA scheme for the cases of average CF-CMI and 6/12-bit CDI. To show the upper bound of the performance, we also plot the performance of the ZF-PA scheme with perfect CSI. As can be seen from Fig. 8, the proposed MALC-PA and RALC-PA schemes achieve considerably larger weighted sum-rate than the ZF-PA scheme when the SNR is medium to high, and their performance approaches that of the ZF-PA scheme with perfect CSI at a relatively fast pace. It should be noted that the RALC-PA scheme shows its advantage over the MALC-PA scheme in all SNR regimes. This is because that the interference minimization strategy is generally not an optimal one and tolerating an appropriate 


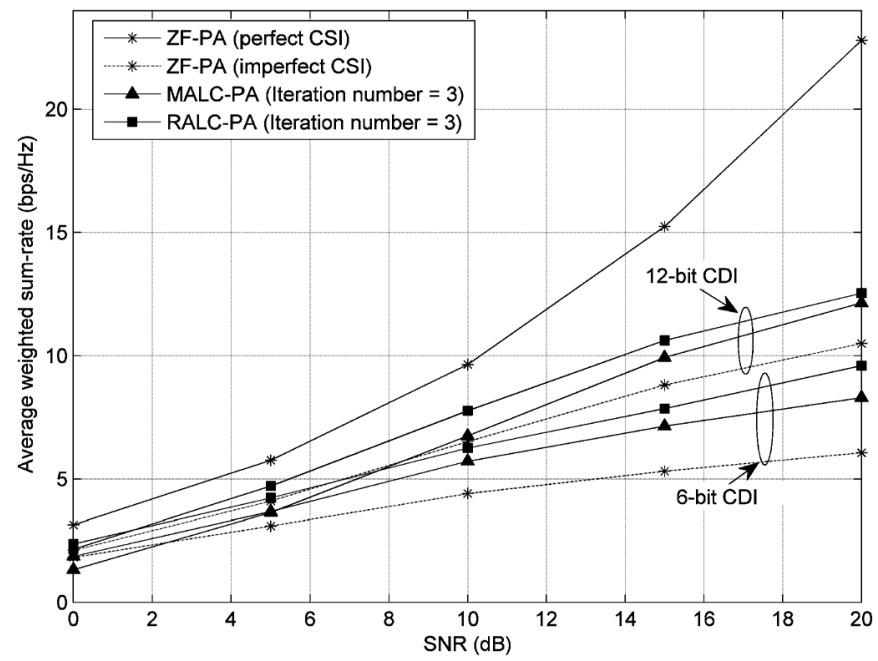

Fig. 8. Average weighted sum-rate performance with per-antenna power constraints.

amount of inter-UE interference with proper UE power allocation is beneficial to optimize the performance objective. Also it should be noted that $L_{\text {algo } 1}$ is set to 3 because the proposed algorithm converges rather quickly with regard to $L_{\text {algo1 }}$ as have been shown in Fig. 7 .

As future works, closed-form evaluation of the objective function in the optimization problem, analysis on the relationship between the performance and feedback size, the impact of CDI feedback delay, as well as more practical fading channel model and non-RVQ CDI codebook will be considered for the proposed beamforming schemes. In addition, the extensions to more sophisticated systems such as MIMO relay networks and multi-cell cooperative broadcast channels will be investigated in the future.

\section{CONCLUSIONS}

In this paper, leakage-based robust beamforming for MU-MISO system with per-antenna power constraints and quantized CDI is studied. Based on our derived CDF of the leakage power for the non-robust ZF beamforming scheme, we propose the MALC-PA and RALC-PA schemes using a two-stage algorithm to alternately update the per-UE power allocations and beamforming vectors in order to maximize the expected weighted sum-rate performance under per-antenna power constraints. Simulation results show that the proposed schemes can achieve better performance than the ZF-PA scheme in terms of average weighted sum-rate performance.

\section{APPENDIX I}

\section{ProOF OF THEOREM 1}

We decompose $\mathbf{w}_{k}(k \neq j)$ as $\mathbf{w}_{k}=\sqrt{\tilde{P}_{k}} \tilde{\mathbf{w}}_{k}=$ $\sqrt{\tilde{P}_{k}}\left(\beta_{k, j} \hat{\mathbf{h}}_{j}^{\mathrm{H}}+\sqrt{1-\left|\beta_{k, j}\right|^{2}} \mathbf{v}_{k, j}\right)$, where $\beta_{k, j}=\hat{\mathbf{h}}_{j} \tilde{\mathbf{w}}_{k}$ and $\mathbf{v}_{k, j}$ is a unit-norm random vector representing the projection of $\tilde{\mathbf{w}}_{k}$ onto the nullspace of $\hat{\mathbf{h}}_{j}^{\mathrm{H}}$. Then, considering (11), we can take the expectation of $\left|\tilde{\mathbf{h}}_{j}^{\diamond} \mathbf{w}_{k}\right|^{2}$ with respect to $\tilde{\mathbf{h}}_{j}^{\diamond}$ and $\tilde{\mathbf{w}}_{k}$, and get

$\mathbb{E}\left\{\left|\tilde{\mathbf{h}}_{j}^{\diamond} \mathbf{w}_{k}\right|^{2}\right\}$

$$
\begin{aligned}
& =\tilde{P}_{k} \mathbb{E}\left\{(1-Z)\left|\beta_{k, j}\right|^{2}+Z\left(1-\left|\beta_{k, j}\right|^{2}\right)\left|\mathbf{e}_{j}^{\diamond} \mathbf{v}_{k, j}\right|^{2}\right\} \\
& \quad+\tilde{P}_{k} \mathbb{E}\left\{2 \operatorname{Re}\left\{\sqrt{(1-Z) Z\left(1-\left|\beta_{k, j}\right|^{2}\right)} \beta_{k, j} \mathbf{e}_{j}^{\diamond} \mathbf{v}_{k, j}\right\}\right\} \\
& \stackrel{(\text { a })}{=} \tilde{P}_{k} \mathbb{E}\left\{(1-Z)\left|\beta_{k, j}\right|^{2}+Z\left(1-\left|\beta_{k, j}\right|^{2}\right)\left|\mathbf{e}_{j}^{\diamond} \mathbf{v}_{k, j}\right|^{2}\right\} \\
& \stackrel{(\text { b) }}{=} \tilde{P}_{k} \mathbb{E}\left\{(1-Z)\left|\beta_{k, j}\right|^{2}+Z\left(1-\left|\beta_{k, j}\right|^{2}\right) \frac{1}{N-1}\right\} \\
& =\tilde{P}_{k}\left[\frac{\eta}{N-1}+\left(1-\frac{N}{N-1} \eta\right) \mathbb{E}\left\{\left|\beta_{k, j}\right|^{2}\right\}\right] .
\end{aligned}
$$

Equation (a) is obtained because $Z, \beta_{k, j}, \mathbf{e}_{j}^{\diamond}$ and $\mathbf{v}_{k, j}$ are independently distributed. Equation (b) holds because $\mathbf{e}_{j}^{\diamond}$ and $\mathbf{v}_{k, j}^{\mathrm{H}}$ are i.i.d. isotropic vectors located in the $(N-1)$-dimensional nullspace of $\hat{\mathbf{h}}_{j}$. Hence $\left|\mathbf{e}_{j}^{\diamond} \mathbf{v}_{k, j}\right|^{2}$ follows a beta $(1, N-2)$ distribution, and the mean value of which equals to $\frac{1}{N-1}$ [29]. From (14) and (39), we can see that $\mathbb{E}\left\{\left|\tilde{\mathbf{h}}_{j}^{\diamond} \mathbf{w}_{k}\right|^{2}\right\}$ is a monotonically increasing affine function with respect to $\mathbb{E}\left\{\left|\beta_{k, j}\right|^{2}\right\}$. Thus, $\mathbb{E}\left\{\left|\tilde{\mathbf{h}}_{j}^{\diamond} \mathbf{w}_{k}\right|^{2}\right\}$ achieves its minimum value

$$
\min \left(\mathbb{E}\left\{\left|\tilde{\mathbf{h}}_{j}^{\diamond} \mathbf{w}_{k}\right|^{2}\right\}\right)=\frac{\tilde{P}_{k} \eta}{(N-1)},
$$

when $\mathbb{E}\left\{\left|\beta_{k, j}\right|^{2}\right\}=0$. It implies that $\beta_{k, j}=0$ since $\left|\beta_{k, j}\right|^{2} \geq 0$ is always true. On the other hand, $\mathbb{E}\left\{\left|\tilde{\mathbf{h}}_{j}^{\diamond} \mathbf{w}_{k}^{\mathrm{ZF}}\right|^{2}\right\}$ can be calculated as

$$
\begin{aligned}
& \mathbb{E}\left\{\left|\tilde{\mathbf{h}}_{j}^{\diamond} \mathbf{w}_{k}^{\mathrm{ZF}}\right|^{2}\right\} \\
& =\mathbb{E}\left\{\left|\left(\left(\sqrt{1-Z} \hat{\mathbf{h}}_{j}+\sqrt{Z} \mathbf{e}_{j}^{\diamond}\right)\right)\left\|\mathbf{w}_{k}^{\mathrm{ZF}}\right\| \tilde{\mathbf{w}}_{k}^{\mathrm{ZF}}\right|^{2}\right\} \\
& =\tilde{P}_{k} \mathbb{E}\{Z\} \mathbb{E}\left\{\left|\mathbf{e}_{j}^{\diamond} \tilde{\mathbf{w}}_{k}^{\mathrm{ZF}}\right|^{2}\right\} \\
& \stackrel{(\text { a) }}{=} \frac{\tilde{P}_{k} \eta}{(N-1)} .
\end{aligned}
$$

Equation (a) comes from the fact that $\left|\mathbf{e}_{j}^{\diamond} \tilde{\mathbf{w}}_{k}^{\mathrm{ZF}}\right|^{2}$ also conforms to a beta(1,N-2) distribution [6], and the mean value of which is $\frac{1}{N-1}$. Our proof is completed by combining (40) and (41) to form an inequality with multiplication of $\xi_{j}^{2} A_{j}$ to both sides since CMI of UE $j$ is independent of (39)-(41).

\section{APPENDIX II}

PROOF OF THEOREM 2

According to (7) and (11), we can get $\left|\tilde{\mathbf{h}}_{j}^{\diamond} \tilde{\mathbf{w}}_{k}^{\mathrm{ZF}}\right|^{2}=$ $\left|\left(\sqrt{1-Z} \hat{\mathbf{h}}_{j}+\sqrt{Z} \mathbf{e}_{j}^{\diamond}\right) \tilde{\mathbf{w}}_{k}^{\mathrm{ZF}}\right|^{2}=Z\left|\mathbf{e}_{j}^{\diamond} \tilde{\mathbf{w}}_{k}^{\mathrm{ZF}}\right|^{2}$. Let $G=$ $\left|\mathbf{e}_{j}^{\diamond} \tilde{\mathbf{w}}_{k}^{\mathrm{ZF}}\right|^{2}$. Then we define a random variable $V$ as $V=\left|\tilde{\mathbf{h}}_{j}^{\diamond} \tilde{\mathbf{w}}_{k}^{\mathrm{ZF}}\right|^{2}=Z G$. Furthermore, we define a random variable $D$ as $D=R V$, where the random variable $R=A_{j}^{\diamond}$. According to [26], $R$ is chi-square distributed with $2 N$ degrees of freedom, each with variance $1 / 2$. Hence its PDF and CDF can be respectively written as $p_{R}(r)=\frac{1}{\Gamma(N)} r^{N-1} \mathrm{e}^{-r}$ and $P_{R}(r)=1-\mathrm{e}^{-r} \sum_{l=0}^{N-1} \frac{r^{l}}{l !}$. Then, based on the result in Theorem 3, the CDF of $D=R V$ can be derived as (42),

$$
\begin{aligned}
& P_{D}(d) \\
& \quad=\operatorname{Pr}(R V \leq d)
\end{aligned}
$$




$$
\begin{aligned}
= & \operatorname{Pr}\left(V \leq \frac{d}{R}\right) \\
= & \int_{0}^{\infty} P_{V}\left(\frac{d}{r}\right) p_{R}(r) d r \\
= & \int_{0}^{d} p_{R}(r) d r+\int_{d}^{\infty} P_{V}\left(\frac{d}{r}\right) p_{R}(r) d r \\
= & {\left[\frac { 1 } { ( N - 2 ) ! } \left[\sum_{n=0}^{N-2} \sum_{m=1}^{2^{B}} \mathrm{C}_{N-2}^{n} \mathrm{C}_{2^{B}}^{m} \frac{(-1)^{n+m} m d^{n}}{m N-(m+n)}\right.\right.} \\
& \left.\times \frac{1}{(N-2) !} \int_{d}^{\infty} r^{(N-1-n)} \mathrm{e}^{-r} d r\right] \\
& \times \int_{d=0}^{N-2} \sum_{m=1}^{2^{B}} \mathrm{C}_{N-2}^{n} \mathrm{C}_{2^{B}}^{m} \frac{(-1)^{n+m} m d^{m(N-1)}}{m N-(m+n)}
\end{aligned}
$$$$
\stackrel{(\mathrm{a})}{=} 1+\frac{1}{(N-2) !}
$$$$
\times\left\{\sum_{n=0}^{N-2} \sum_{m=1}^{2^{B}} \mathrm{C}_{N-2}^{n} \mathrm{C}_{2^{B}}^{m} \frac{(-1)^{n+m} m d^{n}}{m N-(m+n)}\right.
$$$$
\left.\times\left.\left[-\mathrm{e}^{-r} \sum_{l=0}^{N-1-n} l ! \mathrm{C}_{N-1-n}^{l} r^{N-1-n-l}\right]\right|_{r=d} ^{r=\infty}\right\}
$$$$
-\frac{1}{(N-2) !}
$$$$
\times\left\{\left.\sum_{n=0}^{N-2} \mathrm{C}_{N-2}^{n} \mathrm{C}_{2^{B}}^{1} \frac{(-1)^{n+1} d^{N-1}}{N-1-n}\left[-\mathrm{e}^{-r}\right]\right|_{r=d} ^{r=\infty}\right\}
$$$$
-\frac{1}{(N-2) !}
$$$$
\times\left\{\sum_{n=0}^{N-2} \sum_{m=2}^{2^{B}} \mathrm{C}_{N-2}^{n} \mathrm{C}_{2^{B}}^{m} \frac{(-1)^{n+m} m d^{m(N-1)}}{m N-(m+n)}\right.
$$$$
\times\left[\sum_{l=1}^{(m-1)(N-1)-1}\right.
$$$$
\left.\left.\times \frac{(-1)^{l} \mathrm{e}^{-r}}{l ! \mathrm{C}_{(m-1)(N-1)-1}^{l} r^{(m-1)(N-1)-l}}\right]\left.\right|_{r=d} ^{r=\infty}\right\}
$$$$
-\frac{1}{(N-2) !}
$$$$
\times\left\{\sum_{n=0}^{N-2} \sum_{m=2}^{2^{B}} \mathrm{C}_{N-2}^{n} \mathrm{C}_{2^{B}}^{m} \frac{(-1)^{n+m} m d^{m(N-1)}}{m N-(m+n)}\right.
$$$$
\left.\times \frac{(-1)^{(m-1)(N-1)-1}}{((m-1)(N-1)-1) !} \int_{d}^{\infty} \frac{\mathrm{e}^{-r}}{r} d r\right\} .
$$

in which equation (a) is obtained according to [29], where $\int r^{i} \mathrm{e}^{-r} d r=-\mathrm{e}^{-r} \sum_{l=0}^{i} l ! \mathrm{C}_{i}^{l} r^{i-l},(i \geq 0)$ and $\int r^{-i} \mathrm{e}^{-r} d r=\sum_{l=1}^{i-1} \frac{(-1)^{l} \mathrm{e}^{-r}}{l ! \mathrm{C}_{i-1}^{l} r^{i-l}}+\frac{(-1)^{i-1}}{(i-1) !} \int \frac{\mathrm{e}^{-r}}{r} d r,(i>0)$. With some mathematical manipulations on (42), we can get the final form of $P_{D}(d)$ shown in (25) of Theorem 2.

\section{APPENDIX III}

PROOF OF THEOREM 3

According to the definitions in Appendix II, $V=$ $\left|\tilde{\mathbf{h}}_{j}^{\diamond} \tilde{\mathbf{w}}_{k}^{\mathrm{ZF}}\right|^{2}=Z G$, where $G=\left|\mathbf{e}_{j}^{\diamond} \tilde{\mathbf{w}}_{k}^{\mathrm{ZF}}\right|^{2}$. According to [6], the probability density function (PDF) and CDF of $Z$ can be expressed as

$p_{Z}(z)=(N-1) \sum_{m=1}^{2^{B}} \mathrm{C}_{2^{B}}^{m}(-1)^{m-1} m z^{m(N-1)-1},(z \in[0,1])$,

and

$$
\begin{aligned}
P_{Z}(z) & =1-\left(1-z^{N-1}\right)^{2^{B}} \\
& =-\sum_{m=1}^{2^{B}} \mathrm{C}_{2^{B}}^{m}(-1)^{m} z^{m(N-1)},(z \in[0,1]) .
\end{aligned}
$$

As explained earlier, $G$ follows a beta $(1, N-2)$ distribution since $\mathbf{e}_{j}^{\diamond}$ and $\left(\tilde{\mathbf{w}}_{k}^{\mathrm{ZF}}\right)^{\mathrm{H}}$ are i.i.d. isotropic vectors in the $(N-$ 1)-dimensional nullspace of $\hat{\mathbf{h}}_{j}$ [6]. Thus, the CDF of $G$ is

$P_{G}(g)=1-(1-g)^{N-2}=-\sum_{n=1}^{N-2} \mathrm{C}_{N-2}^{n}(-1)^{n} g^{n},(g \in[0,1])$.

Therefore, the CDF of $V=Z G$ can be deduced as (46),

$$
\begin{aligned}
P_{V}(v)= & \operatorname{Pr}(Z G \leq v) \\
= & \operatorname{Pr}\left(G \leq \frac{v}{Z}\right) \\
= & \int_{0}^{1} P_{G}\left(\frac{v}{z}\right) p_{Z}(z) d z \\
= & \int_{0}^{v} p_{Z}(z) d z+\int_{v}^{1} P_{G}\left(\frac{v}{z}\right) p_{Z}(z) d z \\
= & P_{Z}(v)+\int_{v}^{1}\left[-\sum_{n=1}^{N-2} \mathrm{C}_{N-2}^{n}(-1)^{n}\left(\frac{v}{z}\right)^{n}\right] \\
& \times\left[-(N-1) \sum_{m=1}^{2^{B}} \mathrm{C}_{2^{B}}^{m}(-1)^{m} m z^{m(N-1)-1}\right] d z \\
= & P_{Z}(v)+(N-1) \sum_{n=1}^{N-2} \sum_{m=1}^{2^{B}} \mathrm{C}_{N-2}^{n} \mathrm{C}_{2^{B}}^{m}(-1)^{n+m} m v^{n} \\
& \times\left.\left[\frac{z^{m N-(m+n)}}{m N-(m+n)}\right]\right|_{z=1} ^{z=1} \\
= & 1+(N-1) \sum_{n=0}^{N-2} \sum_{m=1}^{2^{B}} \mathrm{C}_{N-2}^{n} \mathrm{C}_{2^{B}}^{m}(-1)^{n+m} m \\
& \times \frac{v^{n}-v^{m(N-1)}}{m N-(m+n)} .
\end{aligned}
$$

which concludes our proof. 


\section{REFERENCES}

[1] D. Gesbert, M. Kountouris, R. Heath, C. Chae, and T. Salzer, "From single-user to multiuser communications: Shifting the MIMO paradigm," IEEE Signal Process. Mag., vol. 24, no. 5, pp. 36-46, Sep. 2007.

[2] G. Caire and S. Shamai, "On the achievable throughput of a multiantenna Gaussian broadcast channel," IEEE Trans. Inf. Theory, vol. 49, no. 7, pp. 1691-1706, Jul. 2003.

[3] T. Yoo and A. Goldsmith, "On the optimality of multianntenna broadcast scheduling using zero-forcing beamforming," IEEE J. Sel. Areas Commun., vol. 24, no. 3, pp. 528-541, Mar. 2006.

[4] M. Sharif and B. Hassibi, "A comparison of time-sharing, DPC, and beamforming for MIMO broadcast channels with many users," IEEE Trans. Commun., vol. 55, no. 1, pp. 11-15, Jan. 2007.

[5] Evolved Universal Terrestrial Radio Access (E-UTRA): Physical Layer Procedures 3GPP TS 36.213 V10.0.1, Mar. 2011, Tech. Spec.

[6] N. Jindal, "MIMO broadcast channels with finite-rate feedback," IEEE Trans. Inf. Theory, vol. 52, no. 11, pp. 5045-5060, Nov. 2006.

[7] M. Botros and T. Davidson, "Convex conic formulations of robust downlink precoder designs with quality of service constraints," IEEE J. Sel. Topics Signal Process., vol. 1, no. 4, pp. 714-724, Dec. 2007.

[8] Y. Huang and D. Palomar, "Rank-constrained separable semidefinite programming with applications to optimal beamforming," IEEE Trans. Signal Process., vol. 58, no. 2, pp. 664-678, Feb. 2010.

[9] C. Zhang, W. Xu, and M. Chen, "Robust MMSE beamforming for multiuser MISO systems with limited feedback," IEEE Signal Process. Lett., vol. 16, no. 7, pp. 588-591, Jul. 2009.

[10] M. Sadek, A. Tarighat, and A. Sayed, "A leakage-based precoding scheme for downlink multi-user MIMO channels," IEEE Trans. Wireless Commun., vol. 6, no. 5, pp. 1711-1721, May 2007.

[11] B. Dai, W. Xu, and C. Zhao, "Multiuser beamforming optimization via maximizing modified SLNR with quantized CSI feedback," in Proc. IEEE Wireless Commun., Netw. Mobile Comput. (WiCOM 2011), Sep. 2011, pp. 1-5.

[12] H. Du and P. Chung, "A probabilistic approach for robust leakage-based MU-MIMO downlink beamforming with imperfect channel state information," IEEE Trans. Wireless Commun., vol. 11, no. 3, pp. 1239-1247, Mar. 2012.

[13] P. Chung and H. Du, "Robust SLNR downlink beamforming based on Markov's inequality," in Proc. IEEE Int. Conf. Commun. (ICC 2012), Jun. 2012, pp. 3680-3684.

[14] K. Huang and R. Zhang, "Cooperative precoding with limited feedback for MIMO interference channels," IEEE Trans. Wireless Commun., vol. 11, no. 3, pp. 1012-1021, Mar. 2012

[15] M. Joham, P. Castro, W. Utschick, L. Castedo, and L. , "Robust precoding with limited feedback design based on precoding MSE for MU-MISO systems," IEEE Trans. Signal Process., vol. 60, no. 6, pp. 3101-3111, Jun. 2012.

[16] J. Zou, W. Liu, M. Ding, H. Luo, and H. Yu, "Transceiver design for AF MIMO two-way relay systems with imperfect channel estimation," in Proc. IEEE Global Telecommun. Conf. (GLOBECOM 2011), Dec. 2011, pp. 1-5.

[17] Z. Wang, W. Chen, and J. Li, "Efficient beamforming for MIMO Relaying broadcast channel with imperfect channel estimation," IEEE Trans. Veh. Technol., vol. 61, no. 1, pp. 419-426, Jan. 2012.

[18] Z. Wang, W. Chen, F. Gao, and J. Li, "Capacity performance of efficient relay beamformings for dual-hop MIMO multi-relay networks with imperfect R-D CSI at relays," IEEE Trans. Veh. Technol., vol. 60, no. 6, pp. 2608-2619, Jun. 2011.

[19] A. Tajer, N. Prasad, and X. Wang, "Robust linear precoder design for multi-cell downlink transmission," IEEE Trans. Signal Process., vol. 59, no. 1, pp. 235-251, Jan. 2011.

[20] W. Yu and T. Lan, "Transmitter optimization for the multi-antenna downlink with per-antenna power constraints," IEEE Trans. Signal Process., vol. 55, no. 6, pp. 2646-2660, Jun. 2007.

[21] H. Wan, W. Chen, and J. Ji, "Efficient linear transmission strategy for MIMO relaying broadcast channels with direct links," IEEE Wireless Commun. Lett., vol. 1, no. 1, pp. 14-17, Jan. 2012

[22] M. Ding, J. Zou, Z. Yang, H. Luo, and W. Chen, "Sequential and incremental precoder design for joint transmission network MIMO systems with imperfect backhaul," IEEE Trans. Veh. Technol., vol. 61, no. 6, pp. 2490-2503, Jul. 2012.

[23] Z. Wang and W. Chen, "Regularized zero-forcing for multiantenna broadcast channels with user selection," IEEE Wireless Commun. Lett., vol. 1, no. 2, pp. 129-132, Feb. 2012.

[24] W. Santipach and M. Honig, "Asymptotic capacity of beamforming with limited feedback," in Proc. IEEE Int. Symp. Inf. Theory (ISIT 2004), Jun. 2004, p. 289.

[25] S. Sesia, I. Toufik, and M. Baker, LTE: The UMTS Long Term Evolution. New York: Wiley, 2009.

[26] J. Proakis, Digital Communications, 3rd ed. New York: McGrawHill, 1995.
[27] A. Wiesel, Y. Elda, and S. Shamai, "Zero-forcing precoding and generalized inverses," IEEE Trans. Signal Process., vol. 56, no. 9, pp 4409-4418, Sep. 2008.

[28] M. Grant and S. Boyd, "CVX:Matlab software for disciplined convex programming, vers. 2.0 beta," Sep. 2013 [Online]. Available: http:// cvxr.com/cvx

[29] I. Gradshteyn and I. Ryzhik, Table of Integrals, Series, and Products, 7th ed. New York: Elsevier, 2007

[30] Z. Luo, W. Ma, A. So, Y. Ye, and S. Zhang, "Semidefinite relaxation of quadratic optimization problems," IEEE Signal Process. Mag., vol. 27, no. 3, pp. 20-34, May 2010.

[31] M. Chiang, "Geometric programming for communication systems," Short Monograph in Found. Trends in Commun. Inf. Theory, vol. 2, Jul. 2005.

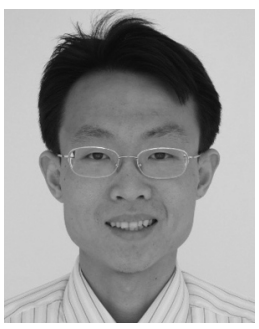

Ming Ding (M'12) received the B.S., M.S. and $\mathrm{Ph} . \mathrm{D}$. degrees in electronics engineering from Shanghai Jiao Tong University in 2004, 2007, and 2011, respectively.

$\mathrm{He}$ is a principal researcher at SHARP Laboratories of China. His research interests include MIMO-OFDM technology, OFDM synchronization, relay systems, interference management, cooperative communications and modeling wireless communication systems. Up to now, he has published about 20 papers in IEEE journals and conferences, as well as a book on cooperative communications. Also, as the first inventor, he has filed more than 30 patent applications on $4 \mathrm{G} / 5 \mathrm{G}$ technologies.

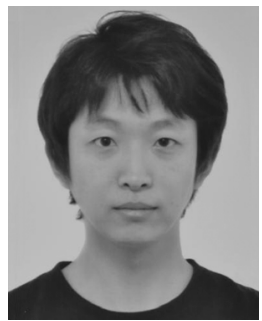

Meng Zhang received the B.S. and M.S. degrees in electronic engineering from Southwest Jiaotong University in 2007 and 2010, respectively.

Currently, he is pursuing his Ph.D. degree at Shanghai Jiao Tong University. His research interests include relay systems, interference mitigation, and cooperative communications.

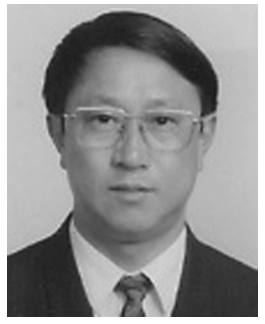

Hanwen Luo was born in 1950. Now he is a professor of Shanghai Jiao Tong University, vice-director of Shanghai Institute of Wireless Communications Technology and vice-director of Institute of Wireless Communication Technology in Shanghai Jiao Tong University. He used to be the leading specialist of China 863 high-tech program on Beyond 3G wireless communication systems and China 973 high-tech program on the researches of military equipment. His research interests include mobile and personal communications.

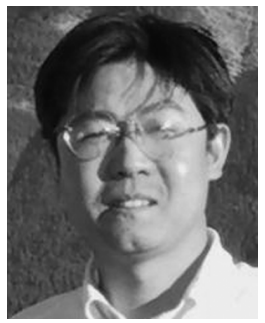

Wen Chen (M'03-SM'11) received the B.S. and M.S. degrees from Wuhan University, China, in 1990 and 1993, respectively, and the Ph.D. degree from the University of Electro-Communications, Tokyo, Japan, in 1999.

He was a researcher of Japan Society for the Promotion of Sciences (JSPS) from 1999 through 2001. In 2001, he joined the University of Alberta, Canada, starting as a Postdoctoral Fellow with the Information Research Laboratory and continuing as a Research Associate in the Department of Electrical and Computer Engineering. Since 2006, he has been a Full Professor in the Department of Electronic Engineering, Shanghai Jiao Tong University, China, where he is also the Director of the Institute for Signal Processing and Systems. His interests cover network coding, cooperative communications, cognitive radio, and MIMO-OFDM systems. 